\section{UNA ESTRATEGIA EN LA BÚSQUEDA DE MATERIALES}

\author{
Miguel Ángel Alario y Franco \\ Universidad Complutense y Real Academia de Ciencias
}

\section{Introducción: Sobre el concepto de Material}

La Historia de la Humanidad se confunde con la búsqueda de materiales que faciliten la estancia de los seres humanos en la Tierra. Sin más que observar nuestra reacción ante una tormenta en el monte o los problemas que enfrenta una ciudad como Nueva York si le cortan el fluido eléctrico por alguna complicación repentina, imaginamos fácilmente lo que debía ser la vida de nuestros antepasados lejanos, del homo sapiens en adelante, para combatir, por ejemplo, la inclemencia del tiempo. Y esto no incluye sino una parte de las dificultades que nuestros antecesores tuvieron que ir superando para llegar a la época actual que, sin lugar a dudas, es la Época de los Materiales, en afortunada frase del Nobel G.P. Thomson, descubridor de la difracción de los electrones por los cristales, y que con ella ampliaba la tradicional división prehistórica: Edad de Piedra, Edad de Bronce, Edad de Hierro..., al mundo moderno.

$Y$ es que en realidad, no existe actividad humana alguna, con la posible excepción de las actividades fisiológicas: respirar, digerir, pensar..., aunque en todas ellas se utilicen materiales biológicos, no existe, decía, ninguna actividad del homo faber en la que los materiales no jueguen un papel predominante. $Y$, si no, pensemos en la sucesión de acciones que realizamos cada día del alba al ocaso, y aún después de él, hasta que nos abraza el sueño, para percatarnos que del despertador -hecho de materiales metálicos, poliméricos y un poco de vidrio-, a la cafetera -también metálica o plástica, o incluso metálica y plástica-, de la toalla -hecha de fibras cada vez menos naturales y más sintéticas- al cerrojo de la puerta que cada día despedimos con una vuelta de llave -también metálicos- o del catalizador -una compleja combinación de tres o cuatro materiales distintos y costosos- del coche al ascensor de la oficina, y así hasta volver a saludar al cerrojo con otra vuelta de llave en sentido contrario, los materiales han estado presentes de manera permanente aunque discreta: en realidad no nos habiamos dado cuenta de ello, como el bien conocido burgués gentilhombre que no sabía que hablaba en prosa, -cursi, pero en prosa-. Y que además han estado ahí hasta que los hemos querido cambiar por otros mejores, o más adaptados a cada necesidad. Ellos no se cansan, nosotros nos cansamos de ellos y los sustituimos².

¿Y que es, pues, un material? Pues aunque quizá haya una definición para cada tratadista, como ocurre con los términos de uso común que de repente se reconocen como algo específico, una posible definición sería la de "sólido útil con alto valor añadido", que recoge simultáneamente su carácter eminentemente práctico y su interés económico. Acerca de esto último es conveniente hcer las siguientes consideraciones:

El arquetipo de material (de sólido útil) en la Industria Electrónica es, desde luego, el silicio.

Con relación a este último, algunos ejemplos pueden darnos una idea de la importancia económica de los materiales en el mundo actual y la que se puede proyectar hacia el futuro. Así por ejemplo, para los materiales de construcción cemento y madera se indica que el mercado va a pasar de 455 millardos de dólares en 2008 a 571 en $2013^{3}$; de la misma manera, el mercado de productos químicos y materiales para la electrónica ha pasado de 22,7 a 34,8 millardos de dólares en los últimos cinco años ${ }^{4}$.

Evidentemente, el monto total del mercado depende de qué materiales se incluyan en la lista, sobre lo que más adelante volveremos.

Pero para profundizar un poco en eso del "sólido útil", conviene recordar que el concepto de material lleva apa- 
rejadas cuatro ideas maestras que, a menudo, recogiendo el común ordenancismo científico, se asocian a los cuatro vértices de un tetraedro, Figura 1, y que revelan las caracteristicas que hay que conocer para poder controlar y alcanzar el objetivo principal de la Ciencia de Materiales: Realizar materiales a medida, lo que en la literatura científica en lengua inglesa se denomina materials tailoring o simplemente tailoring.

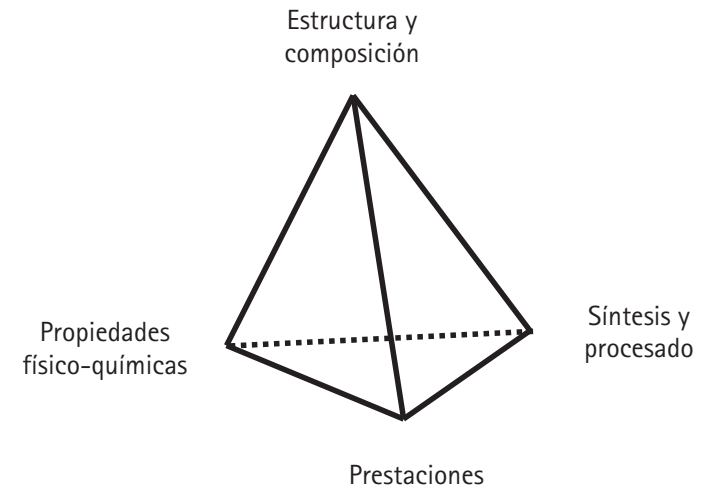

Figura 1. Representación esquemática de los cuatro pilares constitutivos de la Ciencia de Materiales

Hay que recalcar que en este poliedro, como en todos los regulares, los cuatro vértices tienen la misma importancia, aunque se refieren a aspectos distintos. Efectivamente, para conseguir unas buenas prestaciones, por ejemplo un semiconductor de separación entre bandas de $2.0 \mathrm{eV}$, que además resista sin deteriorarse el ataque atmosférico que habitualmente sufre una célula solar fotovoltaica, es necesario conocer la estructura cristalina, y las propiedades fisicoquímicas que aquella y la composición le confieren. Pero también hay que saber fabricar el material: sintetizarlo y procesarlo; esto es, darle la forma adecuada a su utilización concreta. Evidentemente, no es lo mismo utilizar el dióxido de titanio como pigmento blanco para pintar el casco de un yate que como fotocatalizador para la electrolisis del agua. Aunque en ambos casos se trata de $\mathrm{TiO}_{2}$, incluso con la misma estructura, la del mineral rutilo, las condiciones de pureza y tamaño de partícula, entre otras, serían muy diferentes, como diferentes serian sus precios. Y lo mismo podemos decir de una zirconia estabilizada para ladrillos de horno que de otra para células de combustible...
En lo que antecede hemos nombrado ya bastantes materiales, unos por su nombre concreto, por ejemplo zirconia estabilizada, otros por su nombre genérico, por ejemplo metales. Conviene pues tratar de generalizar un poco nuestro concepto de sólido útil y precisar qué materiales incluye. Pero, precisamente debido a la amplitud del término, tampoco están de acuerdo todos los expertos en todas las especies que el género Material debe incluir. Así, se conocen clasificaciones de los materiales basadas en variados criterios: por ejemplo, y esta es quizá la más común, y al mismo tiempo la más sencilla, basados en sus características generales, lo que indica que se trata de clasificarlos a partir de alguna propiedad físico-química específica, y así, se habla de materiales.

Estructurales, que abarcarian: Metales, cerámicas, polímeros, materiales compuestos y vidrios.

Conductores, que incluyen, por lo menos: Sermi y superconductores, conductores iónicos, magnéticos $y_{1}$ por supuesto, los dieléctricos: Ferro-, piezo-, antiferro-, paraeléctricos...

Materiales fotónicos: Ilamados comúnmente materiales ópticos e incluyendo, naturalmente a los magneto-ópticos, electro-ópticos...

\section{Catalizadores, Biomateriales...}

Es evidente, sin embargo, que muchos de estos materiales encajan perfectamente en más de uno de esos grupos, y así se aprecia en los ejemplos, como los dióxidos de zirconio y titanio citados más arriba. Pero es que, además, de este modo, dejamos fuera, nada menos que a los materiales naturales... la madera, las arcillas -que dieron lugar a las cerámicas, decorativas y objetos de culto primero, pero objetos funcionales después-, el carbón en sus formas cristalinas, y a materiales artificiales pero complejos como el cemento...

Adoptando el criterio de tamaños, se habla ahora cada vez más frecuentemente de nanomateriales y nanopartículas, para los que tienen un tamaño del orden de $10^{-9} \mathrm{~m}$, y que están comprendidos entre las dimensiones de las moléculas $(\sim 10 \mathrm{~A})$ y los de las partículas habituales $(\sim 300 \mathrm{~A})$ -aunque en esos límites hay mucho de arbitrario. Pero lo cierto es que en esos tamaños, algunas propiedades se hacen algo diferente debido precisamente a su pequeñez pero también a su grado de interacción. Bien, pues, dentro de ellos ya se recogen términos como los de endo- y exo- 
semiconductores $y$, por otro lado surge la pregunta de a partir de qué tamaño podemos considerar un conjunto de átomos como material 0 , incluso, como sólido, ordenado o no. Pregunta esta que tiene un carácter bastante general y que a veces se "resuelve" en base a la información que sobre dichas partículas pueden dar las técnicas de difracción.

Otras veces, en un afán de abarcar más campo en las clasificaciones, se basan estas en aspectos mucho más generales. Así se han hecho populares clasificaciones que dividen a estos sólidos útiles en dos grandes grupos:

Materiales Moleculares y No-moleculares, o, también, naturales y artificiales incluyendo entre estos a especies tales como el cemento, los aceros, o los materiales compuestos, y entre aquellos a materias tan diversas como la madera, el oro, el diamante o las espinas de los erizos. Parece claro, no obstante que dichas clasificaciones no van muy allá en términos de utilidad de la clasificación...

\section{Estrategia en la búsoueda de Materiales}

A la hora de obtener un nuevo material, es conveniente establecer algún procedimiento sistemático, más allá de la mera combinación de dos o más especies químicas y tratarlas en condiciones adecuadas de presión y temperatura.

Dicho de otro modo: Hay que tener una cierta estrategia, un plan de trabajo cuando se quiere, por ejemplo preparar un semiconductor con una separación entre bandas o zanja de, digamos, $2.5 \mathrm{eV}$. Esa estrategia se puede resumir en cinco apartados que desarrollaremos en lo que sigue:

II.1. Síntesis de Nuevas Composiciones.

II.2. Cambios de estructura a composición constante.

II.3. Cambios de composición manteniendo la estructura

II.4. Materiales compuestos.

II.5. "Otros": Azar.

\section{II.1. Síntesis de Nuevas Composiciones}

Obviamente, la primera manera de preparar nuevos materiales consiste en mezclar diferentes especies químicas y someterlas a algún tratamiento. En general, estos consisten en la utilización, separada o conjunta, de la temperatura y la presión.

Constituye un ejemplo clásico del primer caso la síntesis de la espinela a partir de los óxidos de magnesio y aluminio:

$$
\mathrm{MgO}+\mathrm{Al}_{2} \mathrm{O}_{3} \Rightarrow \mathrm{MgAl}_{2} \mathrm{O}_{4}
$$

Por lo que se refiere al uso de la presión, un ejemplo reciente lo constituye la preparación de clatratos en el sistema metano-agua (Figura 2):

$$
x \mathrm{CH}_{4}+\mathrm{H}_{2} \mathrm{O} \Rightarrow\left(\mathrm{CH}_{4}\right)_{x} \cdot \mathrm{H}_{2} \mathrm{O}
$$

de enorme interés y actualidad por su existencia en los fondos oceánicos y en sistemas planetarios como Saturno y varios de sus satélites.

\section{II.2. Cambios de estructura a composición constante}

Habitualmente, los cambios de estructura sin cambiar la composición, se denominan transiciones de fase; en el caso que nos ocupa se trata, desde luego, de transiciones de fase pero entre dos sólidos con la misma composición y también en este caso pueden ser producidas por presión y/o temperatura. Como veremos en los siguientes ejemplos, las transiciones de fase van acompañadas de cambios sustanciales en las propiedades, lo que genera materiales diferentes.

\section{II.2.1. Transiciones de fase provocadas por la temperatura}

Un buen ejemplo de ellas lo constituye la transformación estructural del dióxido de vanadio de monoclínico a tetragonal, que tiene lugar $a \approx 67^{\circ} \mathrm{C}$, Figura 3: En ella puede observarse que, si bien los átomos de oxígeno solo cambian ligeramente, se produce una importante modificación en las posiciones metálicas que experimentan un notable desplazamiento. Por ello, si en la fase monoclínica existen dos distancias metal-metal, una corta y otra larga (2.65 y $3.12 \mathrm{~A}$, respectivamente) que se pueden interpretar ${ }^{5}$ como debido a la formación de enlaces $\mathrm{V}-\mathrm{V}$ entre cada dos átomos de vanadio, lo que localiza a los electrones de valencia $d^{1}$; en la fase tetragonal, sin embargo, los átomos de vanadio son equidistantes 
Figura 2. Hidratos del metano estudiados por difracción de neutrones bajo presión. El de la derecha contiene una mayor proporción metano/ agua. Círculos rojos oxígeno; círculos violeta carbono; los hidrógenos no están representados. Cortesía del Departamento de cristalografía Universidad de Gotinga

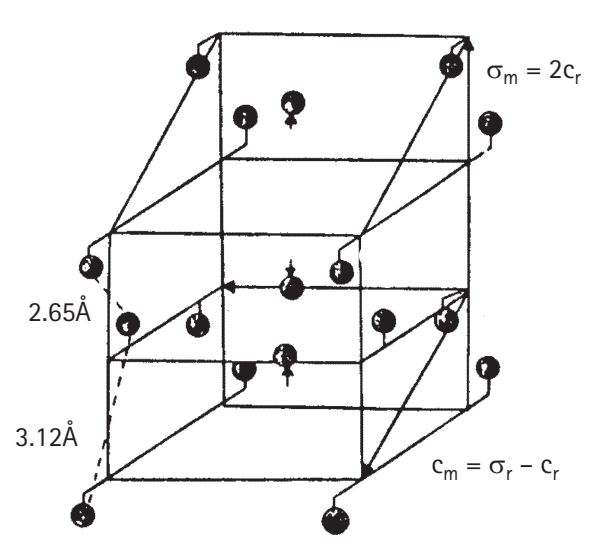

$67^{\circ} \mathrm{C}$
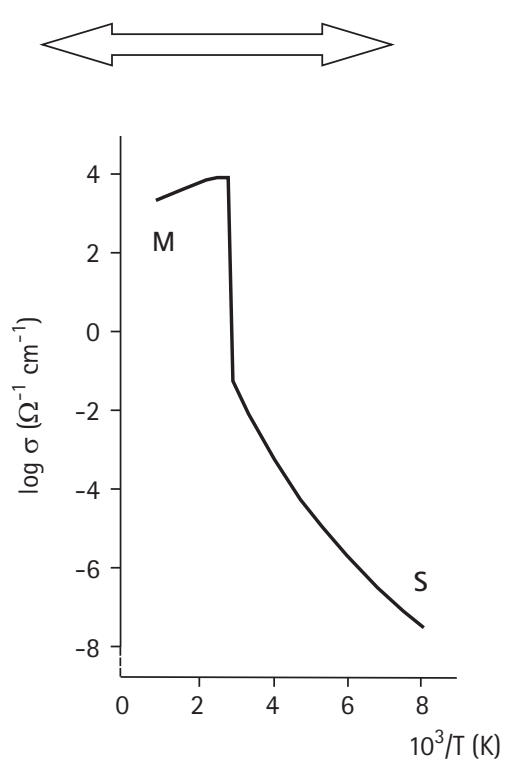

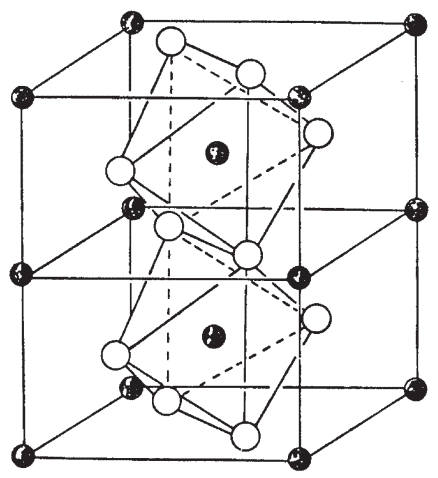

Figura 3. Transición semiconductor(S) - metal(M) en el dióxido de vanadio 
(2.846 A) lo que se interpreta debido a la formación de una banda, de simetría $\delta$, parcialmente ocupada por esos dos electrones $d^{1}$, lo que le confiere propiedades metálicas. En efecto: La resistividad eléctrica del dióxido de vanadio a temperaturas superiores a $67 \mathrm{~K}$ es muy baja, y prácticamente constante: $\rho \approx 8 \times 10^{-4} \Omega \mathrm{cm}$, lo que es característico de los metales. Mientras que a temperaturas inferiores es mucho más alta $\rho \approx 10 \Omega \mathrm{cm}$, y además aumenta al disminuir la temperatura, Figura 3, lo que ocurre en los semiconductores y aislantes. Al mismo tiempo, el material $\mathrm{VO}_{2}$ pasa de diamagnético a paramagnético de Pauli, un estado magnético característico de electrones en una banda de conducción.

No son solo el magnetismo y la conductividad electrónica las propiedades características de los cambios de estructura a composición constante, otros cambios tienen lugar en otras propiedades dependiendo del material en estudio.
Dentro de las propiedades eléctricas, hay otros fenómenos que se ven afectados en el cambio de fase, dando lugar a nuevos materiales. Consideraremos a continuación dos ejemplos bien diferentes.

El primero de ellos es el del ioduro de plata, Agl, del que se conocen dos fases cristalinas que se intercambian a $\approx 145^{\circ} \mathrm{C}$, de una fase tipo wurtzita, $\beta$-Agl, hexagonal en la que por celda unidad hay dos iones $\mathrm{Ag}^{+}$en octaedros de iones $\mathrm{I}^{-}$, a la fase de alta temperatura, $\alpha-\mathrm{Agl}$, donde los iones I- forman un empaquetamiento cúbico centrado en el cuerpo en el que los iones plata se pueden situar en dos de 24 posiciones cristalográficas diferentes ${ }^{6-7}$. Por ello, el desorden presente en los átomos de plata en esa fase da lugar a que presenten una movilidad muy elevada y ello hace que el material $\alpha-\mathrm{Agl}$ sea el mejor conductor iónico de $\mathrm{Ag}^{+}$hasta ahora conocido, Figura 4. Esta propiedad ha sido utilizada en baterías de ión plata.
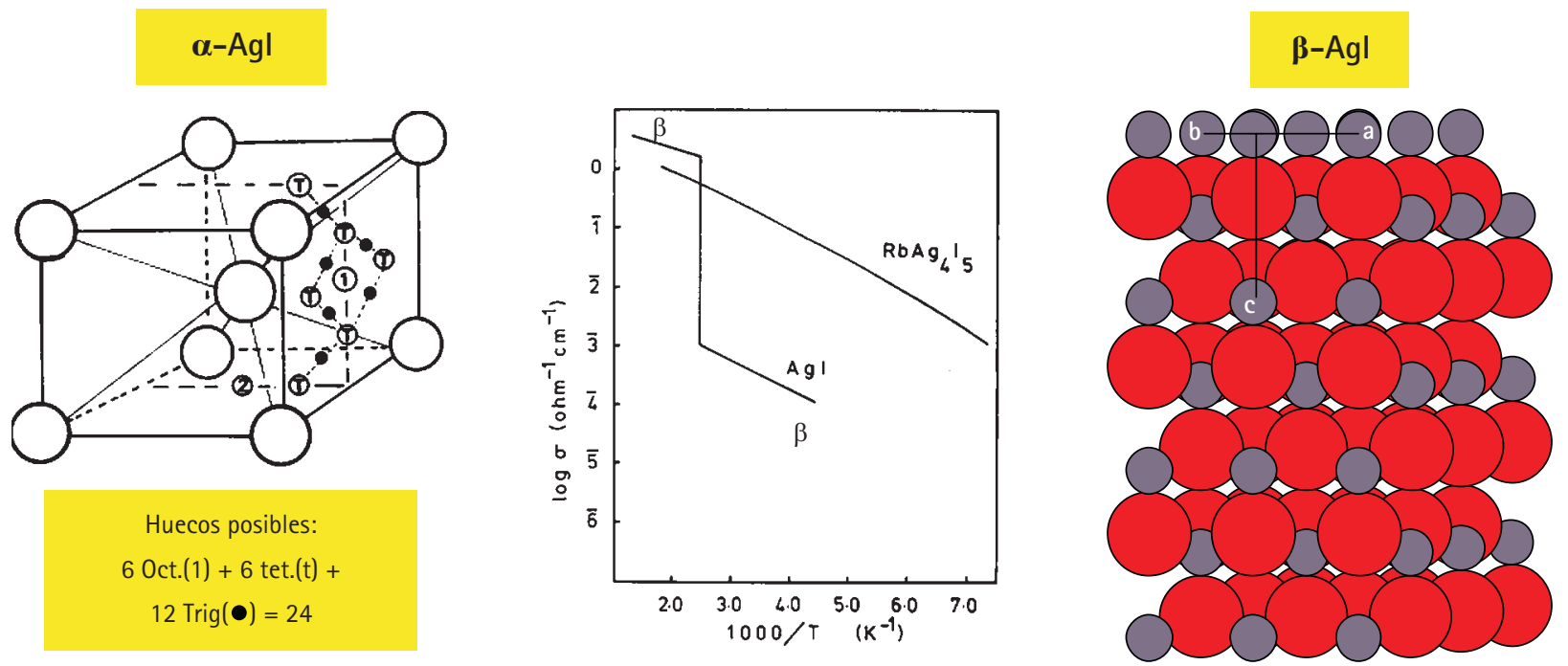

Figura 4. Transición semiconductor $(\beta)$ a conductor iónico $(\alpha)$ en el yoduro de plata.

La figura central recoge la evolución de la conductividad con el inverso de la temperatura

Otro ejemplo muy interesante lo constituye el "titanato de bario" -las comillas corresponden al hecho de que no se trata realmente de una sal bárica de un supuesto ácido titánico, sino de un óxido mixto con importantes propiedades ferroeléctricas, que presenta la estructura perovskita, Figura 5a, o una estructura de ella derivada, una superestructura, dependiendo de la temperatura. Así, pasa sucesivamente por las siguientes fases: Romboédrica, Ortorrómbica, Tetragonal, Cúbica, Hexagonal ${ }^{8}$ y Líquida:

$$
(\mathrm{R}) \stackrel{-80^{\circ} \mathrm{C}}{\longleftrightarrow}(\mathrm{O}) \stackrel{-5^{\circ} \mathrm{C}}{\longrightarrow}(\mathrm{T}) \stackrel{120^{\circ} \mathrm{C}}{\longrightarrow}(\mathrm{C}) \stackrel{1460^{\circ} \mathrm{C}}{\longrightarrow}(\mathrm{H}) \stackrel{1612^{\circ} \mathrm{C}}{\longleftrightarrow} \text { Fundido }
$$


a

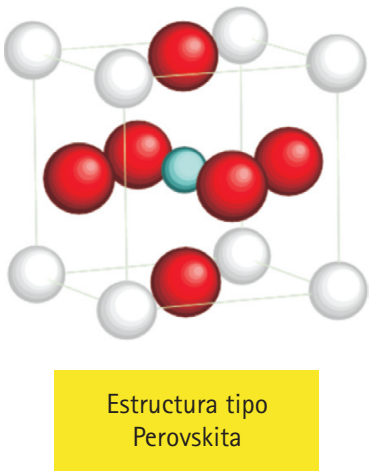

b

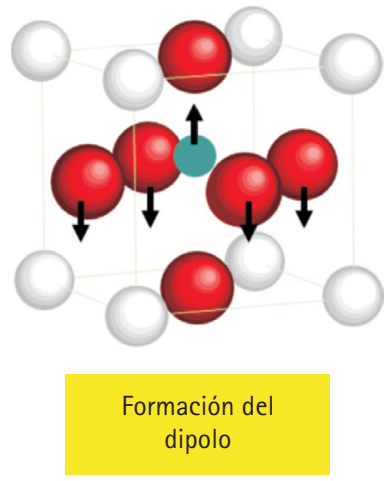

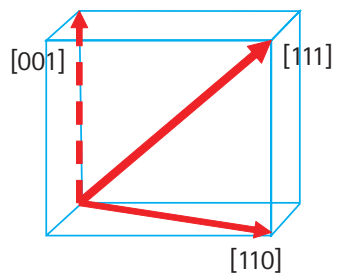

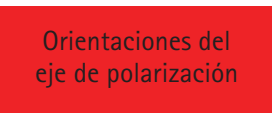

\section{$-80^{\circ} \mathrm{C}$}

$$
-5^{\circ} \mathrm{C}
$$

\section{$120^{\circ} \mathrm{C}$}

Romboédrico(F)

d

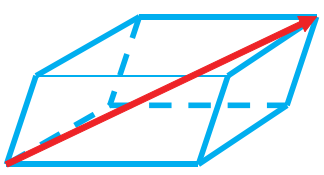

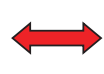

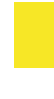

Ortorrómbico (F)

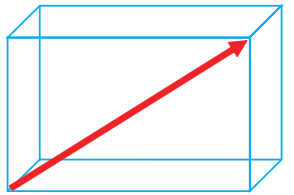

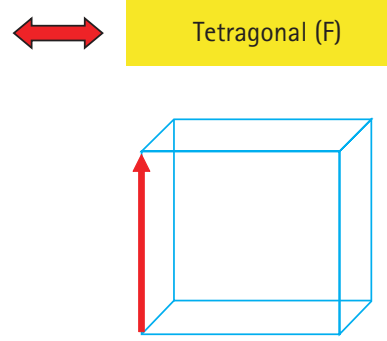

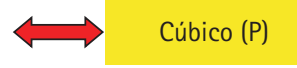

$1460{ }^{\circ} \mathrm{C}$

Figura 5. Transiciones $F \rightarrow F \rightarrow F \rightarrow P$ en el BaTiO ${ }_{3}$ tipo perovskita $(F<>$ Ferroeléctrico; $P<>$ Paraeléctrico)

Efectivamente, a la temperatura ambiente, en realidad entre -5 y $20^{\circ} \mathrm{C}$, la estructura es efectivamente tetragonal, lo que se debe a que, por el elevado tamaño del ión bario, los iones titanio se desplazan, de manera regimentada, y también lo hacen los oxígenos en sentido contrario, Figura $5 b$, del centro del octaedro en la dirección vertical $[001]_{p}$-donde el subíndice $\mathbf{p}$ se refiere a la subestructura perovskita básica- lo que da lugar a un momento dipolar en dicha dirección. Al estar todos los dipolos orientados en la misma dirección, el material es ferroeléctrico con el eje de polarización en la dirección indicada [001]. Pero la orientación del dipolo, Figura $5 c$, depende de la temperatura.

Al bajar la temperatura por debajo de $-5^{\circ} \mathrm{C}$, se produce la reorientación del eje de polarización Figura $5 d$, primero a la dirección [110] y y la estructura se hace ortorrómbica $y$, por debajo de $-80 .{ }^{\circ} \mathrm{C}$ se reorienta en la dirección [111] con lo que la simetría se hace romboédrica, Figura 5c. Dichas transiciones van acompañadas de cambios en la dimensión estructural, Figura $6 a y_{\text {, }}$ más importante aun, en las propiedades dieléctricas. La determinación de la constante dieléctrica en función de la temperatura, Figura $6 b$, refleja claramente la existencia de dichas transiciones y los correspondientes cambios en las propiedades dieléctricas del material. Por otra parte, cuando la temperatura es superior a $120^{\circ} \mathrm{C}$, la agitación térmica de los dipolos hace que estos se sitúen al azar entre todas las direcciones posibles, con lo que el material es en esas condiciones paraeléctrico. 
Figura 6. Evolución de: a) los parámetros reticulares y b) la constante dieléctrica del BaTiO. en función de la temperatura
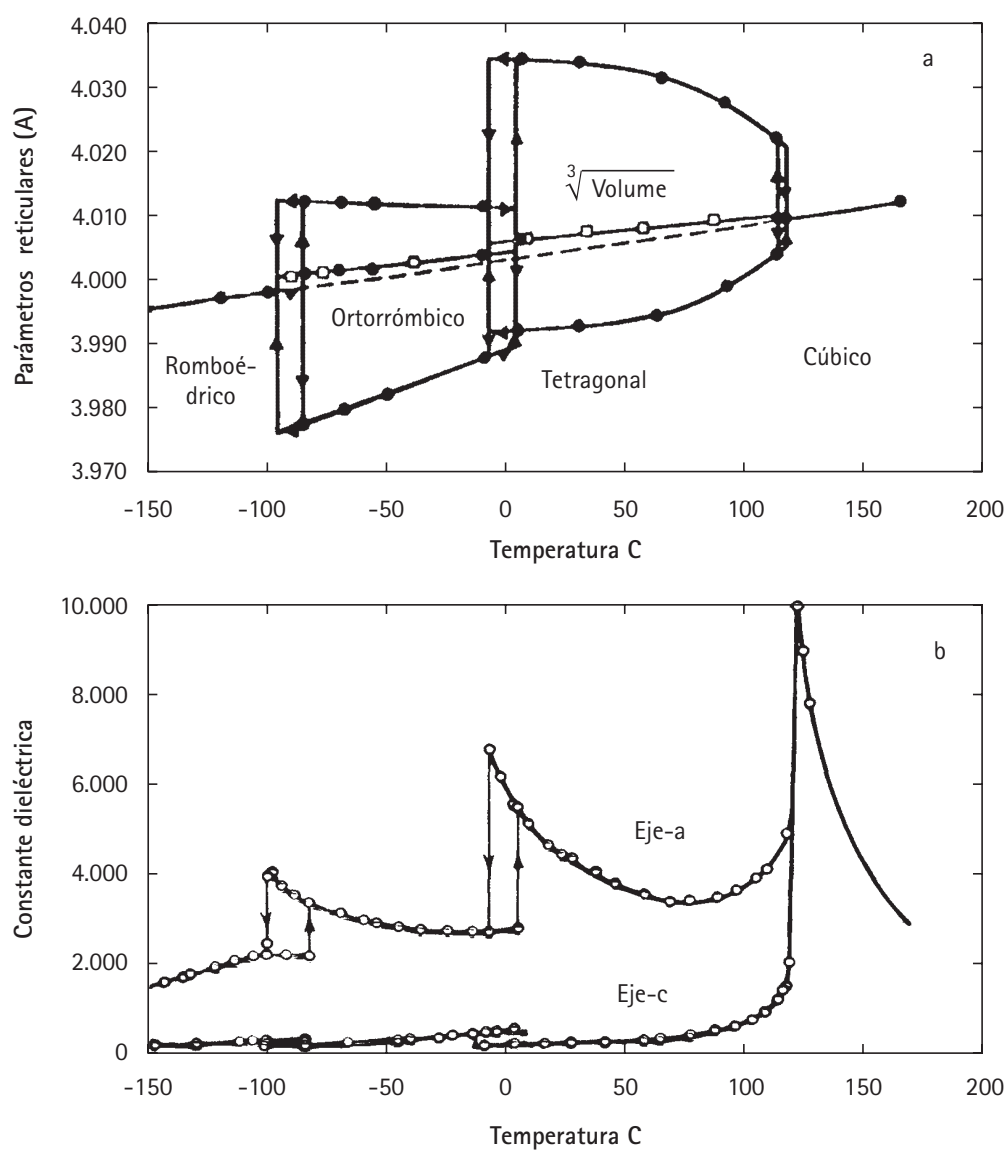

\section{II.2.2. Transiciones de fase provocadas por la presión}

Cuando se aplica presión, y solo presión, a un sólido, a veces se amorfiza. Un ejemplo clásico de ello es el del cuarzo, Figura 7. El proceso de compresión, deforma los enlaces, modifica las distancias interatómicas y el material pierde cristalinidad.
En otras ocasiones, sin embargo, el material sufre una o varias transformaciones de fase. Uno caso reciente de ello es el estudio realizado por Vegas, Jansen et al. ${ }^{9}$ sobre el sulfuro sódico $\mathrm{Na}_{2} \mathrm{~S}$ :

$$
\text { Antifluorita } \stackrel{7 \mathrm{GPa}}{\longrightarrow} \text { Anticotunita }\left(\mathrm{PbCl}_{2}\right) \stackrel{16 \mathrm{GPa}}{\longrightarrow} \text { Tipo }-\mathrm{Ni}_{2} \mathrm{In}
$$

En muchas ocasiones, la transición de fase bajo presión, a temperatura ambiente, va acompañada de importantes cambios en las propiedades. Un ejemplo muy significativo de este comportamiento es el del yodo que es un sólido molecular a presión ambiente y a presiónes del orden de $30 \mathrm{GPa}$ se convierte en un sólido no-molecular metálico en el que ya no se distinguen las moléculas $\mathrm{I}_{2}$, Figura 8.
En ocasiones, se producen cambios en las propiedades en el curso de una transición de fase sin una marcada transformación estructural. Un caso interesante, provocado por la acción conjunta de presión y temperatura, lo constituyen las dos formas cristalinas del sesquióxido de cobalto que cristalizan en la misma estructura, la estructura del mineral corindón. Así, cuando dicho óxido se obtiene a altas presiones y temperaturas:

$$
2 \mathrm{CoF}_{3}+3 \mathrm{Na}_{2} \mathrm{O}_{2} \stackrel{\text { HP\&HT }}{\longrightarrow} 6 \mathrm{NaF}+3 / 2 \mathrm{O}_{2}+\alpha-\mathrm{Co}_{2} \mathrm{O}_{3}
$$




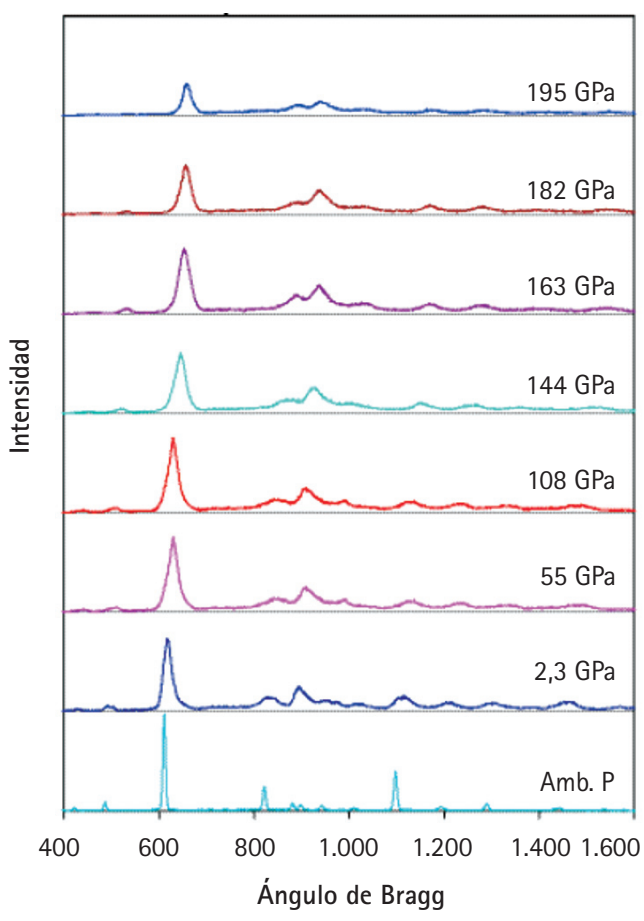

Figura 7. Amorfización progresiva del cuarzo a presiones crecientes y temperatura ambiente

es un material diamagnético, como corresponde a una configuración de bajo espín, $t_{2 g}{ }^{6}$, para el ión Co(III).

Sin embargo cuando este se le calienta a la presión atmosférica

$$
\alpha-\mathrm{Co}_{2} \mathrm{O}_{3} \stackrel{400^{\circ} \mathrm{C} \& \mathrm{P}_{\text {atmoss }}}{\longrightarrow} \beta-\mathrm{Co}_{2} \mathrm{O}_{3}
$$

que es un material paramagnético, como corresponde a una configuración de alto espín $t_{2 q}{ }^{4} e_{g}^{2}$.

Aunque efectivamente existe una ligera variación en el volumen, de 6,7\% a favor de la de alto espín, se conservan el grupo espacial y el tipo estructural. Las propiedades, sin embargo, cambian, se trata pues de un caso de obtención de un nuevo material por cambio de propiedades a estructura y composición constantes. b
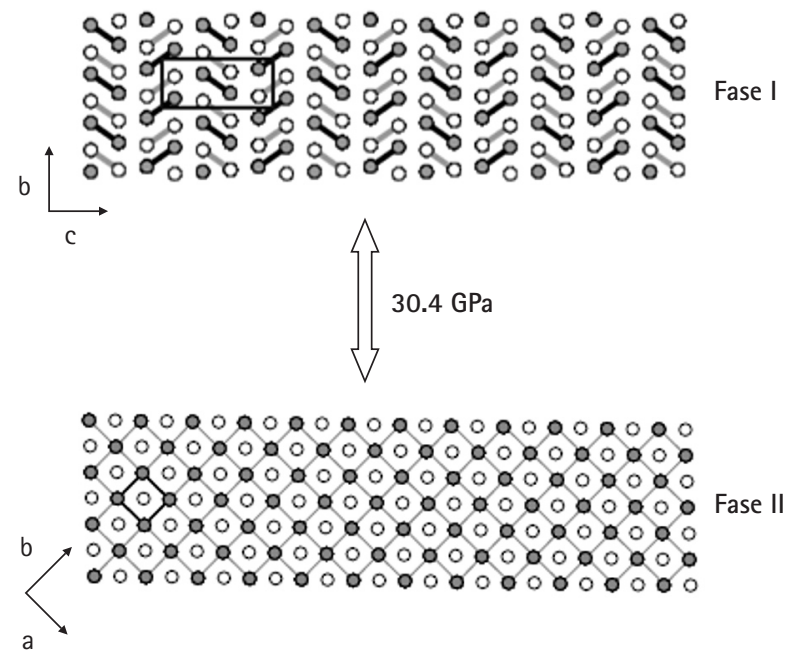

Figura 8. Modificación estructural del yodo bajo presión: paso de un sólido molecular aislante a un sólido no molecular metálico

\section{II.2.3. Transiciones de fase provocadas por presión y temperatura conjuntamente}

De los múltiples ejemplos de este tipo, es sin duda el caso del carbono el mejor conocido. Efectivamente, la búsqueda de diamantes artificiales, sintéticos, fue una carrera memorable en la Química Inorgánica preparativa ${ }^{10}$ y uno de los más tempranos usos industriales de las técnicas de alta presión. Como es de sobra conocido, la estructura laminar del grafito se transforma en la tridimensional del diamante, Figura 9, lo que da lugar a drásticos cambios en las propiedades, Tabla 1.

Tal es este cambio que, junto con otros muchos ejemplos: silicio semiconductor, con la estructura diamante a silicio superconductor con la estructura del $\beta$-Sn, etc. dio lugar a un Paradigma ${ }^{11}$ característico de la Química del Estado Sólido: 

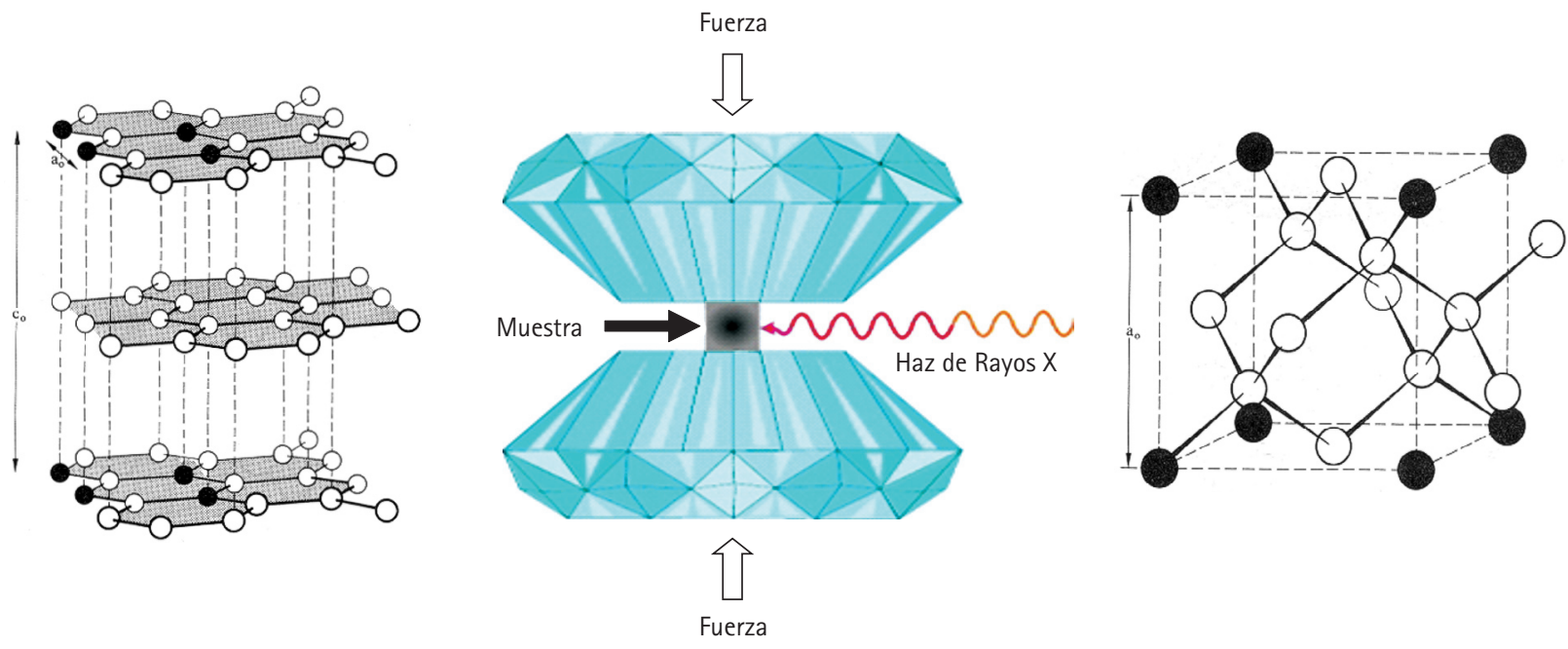

Grafito

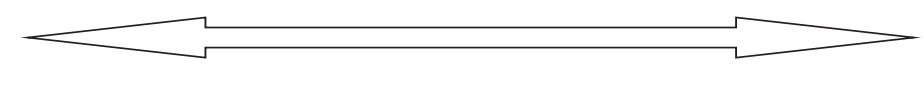

Diamante

$$
\mathrm{C} \text { (Grafito) } \longleftrightarrow \mathrm{C} \text { (Diamante) } \quad \Delta \mathrm{H}=+1.9 \mathrm{~kJ} / \mathrm{M}(300 \mathrm{~K} ; 1 \mathrm{~atm})
$$

Figura 9. Transformación estructural grafito-diamante en la celda yunque de diamante

TABLA I: ALGUNAS DE LAS DIFERENCIAS EXISTENTES ENTRE LAS PROPIEDADES DE LAS FASES GRAFITO Y DIAMANTE DEL CARBONO

\begin{tabular}{|l|c|c|}
\hline & Grafito & Diamante \\
\hline Sistema Cristalino & Hexagonal a $=2.4611 \mathrm{~A} \quad \mathrm{c}=6.7078 \mathrm{~A}$ & 3.514 \\
\hline Densidad $\left(\mathrm{g} / \mathrm{cm}^{3}\right)$ & 2.66 & 10 \\
\hline Dureza $($ Mohs) & $1-2$ & $6 \mathrm{eV}$ \\
\hline Separac. entre bandas & $($ semimetal) & $10^{10}$ \\
\hline$\rho$ eléctrica $(\Omega \mathrm{cm})$ & basal $4 \times 10^{-5} ;$ perp. 0.2 & $-50 ;\{100(50 \mathrm{~K})\}$ \\
\hline$\sigma$ térmica(Tamb.) $(\mathrm{W} / \mathrm{cmK})$ & 15 & 5.87 \\
\hline Constante dieléctrica & basal: $2.61 ;$ perp. 3.28 & 2.41 \\
\hline İnd. refracción $(\lambda=546 \mathrm{~nm})$ & $2.15 ; 1.81$ & \\
\hline
\end{tabular}

Primer paradigma: Relaciones Estructura/Propiedades ${ }^{12}$, QUE REFLEJA LA CLARA Y ESTRECHA RELACIÓN ENTRE UNA Y OTRAS

Sin embargo, existen multitud de ejemplos que muestran que, aun válido, dicho paradigma es insuficiente, incompleto, pues hay montones de compuestos isoestructurales, que constituyen familias de materiales que presentan la misma estructura y no poseen la mismas propiedades (ver más adelante, sección II-3-1).

De entre ellos podemos destacar un par de ejemplos:

Así, en el caso del mineral espinela, el óxido mixto $\mathrm{MgAl}_{2} \mathrm{O}_{4}$ un compuesto de $\mathrm{Mg}(\mathrm{II})$ y $\mathrm{AI}(\mathrm{III})$, es diamagnético, aislante

ARBOR CLXXXVII EXTRA 2011 57-79 ISSN: 0210-1963 
eléctrico e incoloro, mientras que el mineral magnetita $\mathrm{Fe}_{3} \mathrm{O}_{4}$, un óxido mixto de $\mathrm{Fe}(\mathrm{II})$ y $\mathrm{Fe}(\mathrm{III})$ con la misma estructura, es negro, conductor metálico y ferrimagnético.

También, en la familia del rutilo, un conjunto de interesantes materiales que cristalizan en una estructura tetragonal centrada en el cuerpo de átomos metálicos, que ocupan el centro de octaedros más o menos regulares, el dióxido de titanio $\mathrm{TiO}_{2}$, uno de cuyos polimorfos adopta esa estructura y da nombre a la familia, es incoloro, diamagnético y aislante eléctrico. Sin embargo, el dióxido de cromo, $\mathrm{CrO}_{2}$, con la misma estructura es negro, ferromagnético y metálico.

Este tipo de resultados entre muchos otros, como los muy estudiados en la familia de las perovskitas, sugieren que el anterior paradigma es incompleto. Resulta claro que la naturaleza de los átomos que intervienen en la estructura es decisiva a la hora de determinar las propiedades. Por ello, resulta imprescindible extender el paradigma:

Segundo paradigma:

Relaciones Composición/Estructura/Propiedades

No obstante existe otro aspecto de los sólidos que puede influir sobremanera en las propiedades: la Microestructura del Material.

Si bien el concepto de estructura es claro y bien conocido en la Ciencias del Estado Sólido: La disposición de las partes de un objeto o un organismo y, en concreto: Ios componentes y manera de distribuir los átomos en una molécula $o$ en un sólido, el concepto de microestructura es menos frecuentemente tenido en cuenta con la posible excepción de la Metalurgia o la Cerámica...

Por microestructura se entiende a la estructura microscópica de un material o de un organismo y se refiere más a la estructura local, que suministran las técnicas microscópicas que a la estructura media -promediada espacial y temporalmente- que proporcionan los procedimientos difractométricos: neutrones, Rayos $X$ o electrones. De ahí que, en términos generales, se suele decir que la microestructura es la estructura que revela un microscopio ${ }^{13}$. Ciertamente que esa información local depende sobremanera del microscopio que se utiliza, ya que en última instancia, la capacidad de observación de esa estructura local viene dada por la resolución del microscopio que, a su vez depende de la longitud de onda de la luz incidente.

El estudio de la microestructura revela su gran importancia en las propiedades mecánicas de aceros, aleaciones y cerámicas. Sin embargo, no son solo esas propiedades de los materiales las que se ven afectadas por la microestructura.

Así, en la Figura 10, se recoge una fotografía de microscopía electrónica de barrido en la que se observa una película de $\mathrm{YBa}_{2} \mathrm{Cu}_{3} \mathrm{O}_{7}$, producida por electrodeposición de los tres metales sobre un substrato de titanato de estroncio ligeramente reducido y posterior oxidación de los mismos. Las vistas lateral a) y cenital b) muestran la presencia de gruesos filamentos verticales, tipo espárragos, formados por pequeñas partículas del material y medidas magnéticas ${ }^{14}$ muestran inequívocamente que se trata de un material superconductor. Sin embargo es evidente que si colocamos unos electrodos a ambos lados de la muestra no podemos pasar una corriente eléctrica por la falta de continuidad entre los cristales, esa microestructura impide pues la utilización de ese material en un dispositivo superconductor.

La naturaleza de la microestructura se pone también de manifiesto en la Figura 11a, que muestra, por medio de microscopía electrónica de transmisión, la presencia de defectos extensos bidimensionales -las líneas blancas paralelas, pero sin correlación transnacional, que aparecen en la misma- en otra preparación del mencionado superconductor $^{15}$. Un ejemplo adicional, a mayor resolución, se muestra en la Figura $11 \mathrm{~b}$, en la que una preparación supuestamente de $\operatorname{PrBa}_{2} \mathrm{Cu}_{3} \mathrm{O}_{7}$, resulta ser $^{16}$ un intercrecimiento de dos fases muy próximas. La mencionada y el óxido $\mathrm{PrBa}_{2} \mathrm{Cu}_{4} \mathrm{O}_{7,5}$. Cabe señalar que esas microestructuras locales escapan detección a las técnicas no microscópicas. De ahí que como dijo D. Wadsley, "la estructura de un material depende de longitud de onda con que se le observe".

Resumiendo: La microestructura incluye además de la estructura, los defectos, y el desorden, así como la morfología del material.

Así pues, tenemos que establecer un tercer y, por ahora, último paradigma: 

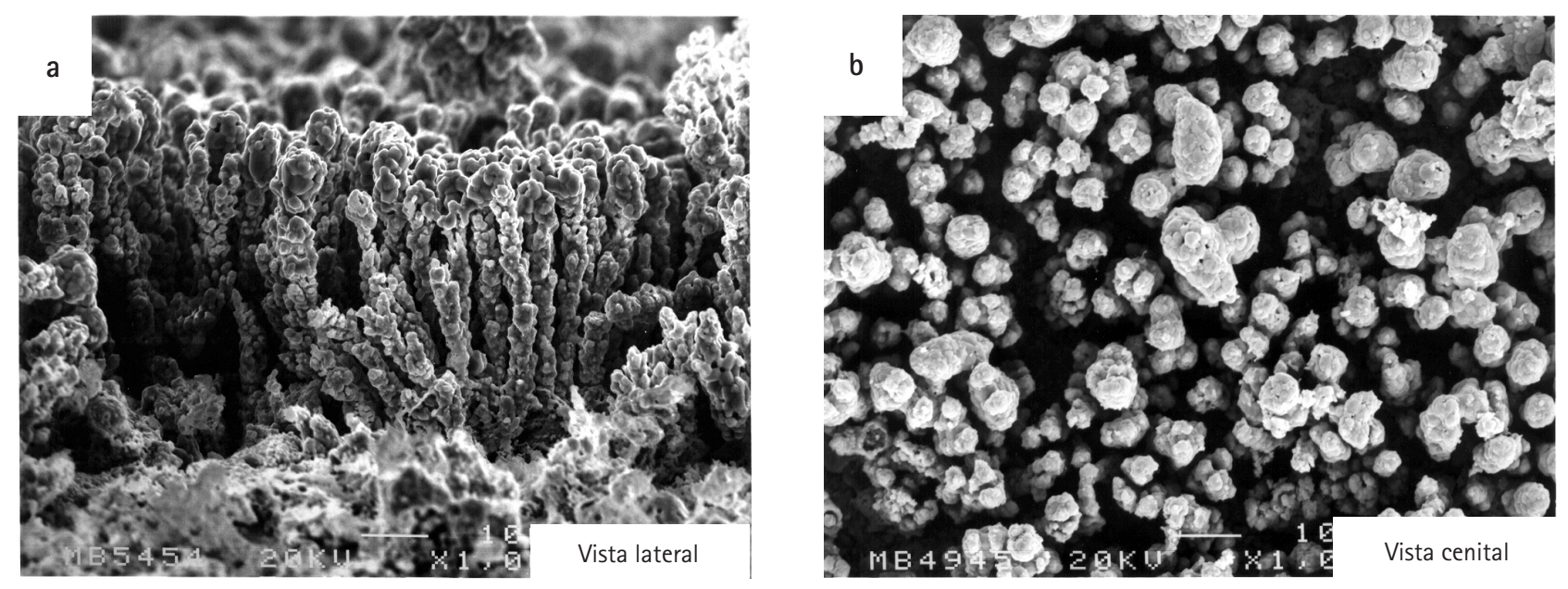

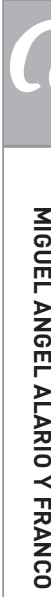

Figura 10. Imágenes de Microscopía Electrónica de Barrido del superconductor $\mathrm{YBa}_{2} \mathrm{Cu}_{3} \mathrm{O}_{7-x}$ depositado sobre $\mathrm{SrTiO}_{3}$
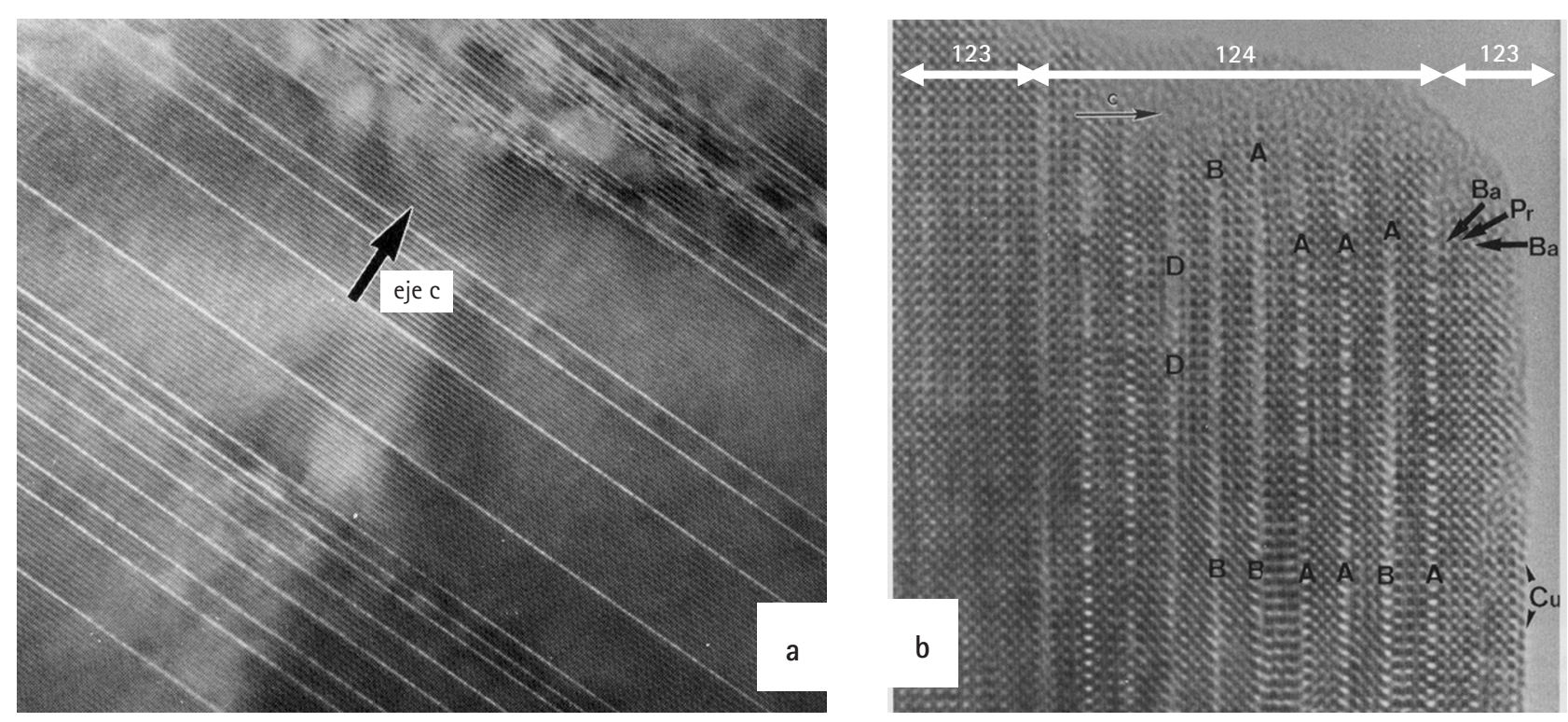

Figura 11. Imágenes de Microscopía electrónica de transmisión, mostrando: a) defectos extensos paralelos al eje c en el superconductor $\mathrm{YBa}_{2} \mathrm{Cu}_{3} \mathrm{O}_{7 . x}$; b) Intercrecimiento de dos óxidos mixtos de cobre $123\left(\mathrm{PrBa}_{2} \mathrm{Cu}_{3} \mathrm{O}_{7 . x}\right)$ and $124\left(\mathrm{PrBa}_{2} \mathrm{Cu}_{4} \mathrm{O}_{4}\right)$

Tercer paradigma: Relaciones Composición/Estructura/ Microestructura/Propiedades

Cabe señalar, para terminar este apartado, que la nanociencia y la nanotecnología aprovechan las excepcionales propiedades de la materia a la escala microscópica, su microestructura -que ya anticipó Richard Feynman.

doi: 10.3989/arbor.2011.extran1114
II.3. Cambios de composición manteniendo la estructura

A la hora de estudiar las modificaciones que en las propiedades de los materiales tiene una variación en la composición de un sólido en una estructura cristalina determinada, es conveniente diferenciar dos casos ARBOR CLXXXVII EXTRA 2011 57-79 ISSN: 0210-1963 
según que se mantenga o no el número de posiciones ocupadas:

II.3.1. Cambio de todos los átomos de un tipo manteniendo constante el número de sitios ocupados. Así se generan familias de compuestos ${ }^{17}$.

II.3.2. Cambio de algunos átomos de un tipo manteniendo constante el número de sitios ocupados. Esto da lugar a soluciones sólidas verdaderas.

II.3.3. Cambio de algunos átomos de un tipo cambiando el número de sitios ocupados. Así se generan soluciones sólidas anómalas o compuestos no estequiométricos ${ }^{18}$.

II.3.1. Cambio de todos los átomos de un tipo manteniendo constante el número de sitios ocupados

Existen pocas estructuras cristalinas que sean monocomposicionales y el fenómeno más común, tanto en el mundo natural como en el artificial o sintético, es la existencia de un gran número de especies químicas isoestructurales.

Como ya se recogía en la descripción del segundo paradigma y por extensión en el tercero, cuando dos compuestos son isoestructurales tienen propiedades diferentes y constituyen materiales distintos aunque relacionados y miembros de una misma familia. Así por ejemplo, en la familia del Rutilo, descrita por la estructura cristalina de una de las fases del dióxido de titanio, $\mathrm{TiO}_{2}$, existen materiales semiconductores, metálicos, ferro-, dia- y paramagnéticos, coloreados o transparentes-incoloros-, etc., Tabla II. Una situación similar se da en la familia de la perovskita, Figura 12 y Tabla III, y en muchas otras familias: monóxidos con el tipo estructural del cloruro sódico ${ }^{19}$, la familia de los granates ${ }^{20}$ o la de de las ferri$\operatorname{tas}^{21}$ : un subgrupo de la familia de las espinelas y un muy larguísimo etcétera.

TABLA II: PROPIEDADES DE ALGUNOS MIEMBROS DE LA FAMILIA DEL RUTILO

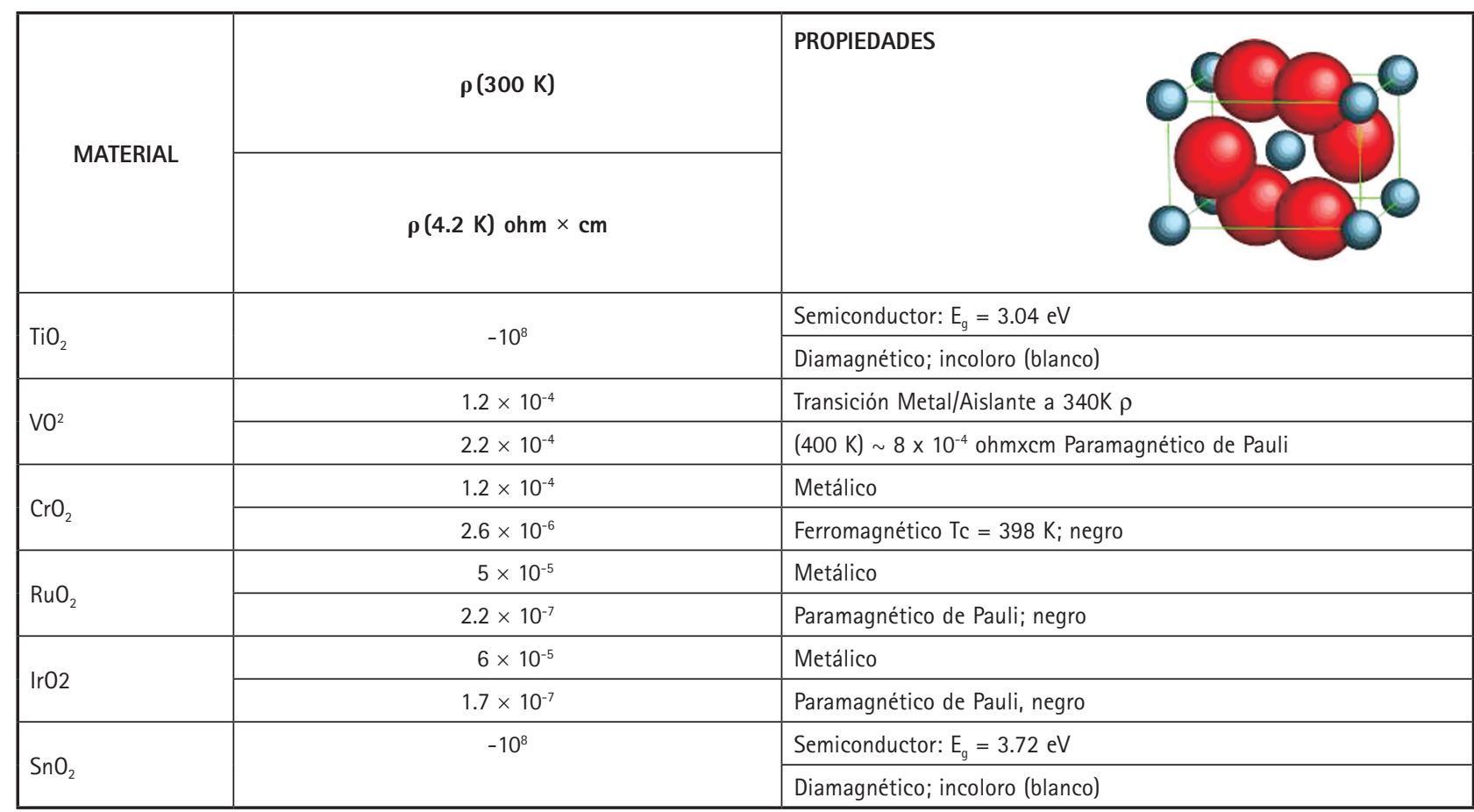




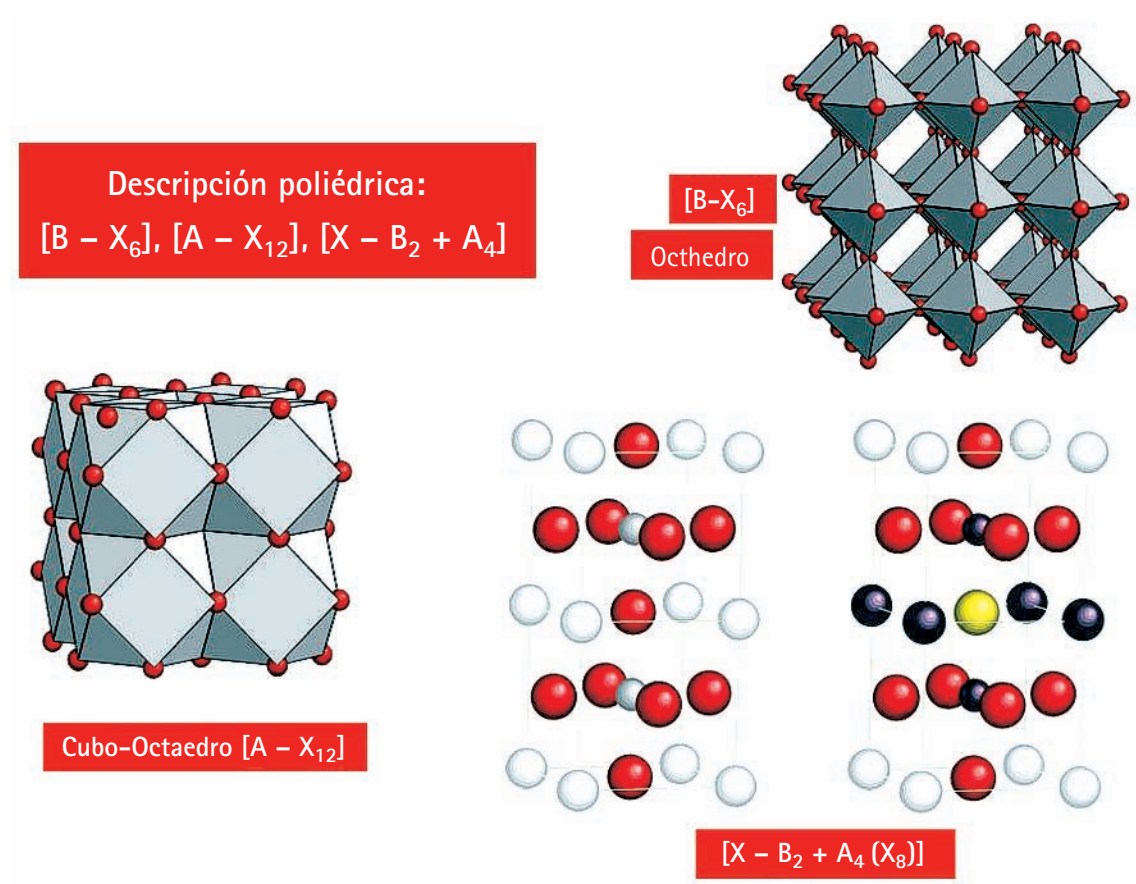

Figura 12. Estructura de la perovskita: descripción poliédrica

TABLA III: PROPIEDADES DE ALGUNOS MIEMBROS DE LA FAMILIA DE LA PEROVSKITA

\begin{tabular}{|c|c|c|}
\hline MATERIAL & SIMETRÍA & PROPIEDAD CARACTERÍSTICA \\
\hline $\mathrm{SrTiO}_{3}$ & Cúbico & Semiconductor: $\mathrm{E}_{g}=3.0 \mathrm{eV}$ \\
\hline $\mathrm{K}_{\mathrm{x}} \mathrm{WO}_{3}$ & Cúbico & Metal. Paramag. Pauli \\
\hline $\mathrm{Tl}_{x} \mathrm{WO}_{3}$ & hexagonal & Superconductor; $\mathrm{Tc}=2.1 \mathrm{~K}$ \\
\hline $\mathrm{BaTiO}_{3}$ & Tetragonal & Ferroeléctrico \\
\hline $\mathrm{PbZrO}_{3}$ & Ortorrómbico & Antiferroeléctrico \\
\hline $\mathrm{Pb}\left(\mathrm{Zr}_{0.65} \mathrm{TiO}_{0.35}\right) \mathrm{O}_{3}$ & Romboédrico & Piezoeléctrico \\
\hline $\mathrm{Fe}_{3} \mathrm{FeN}$ (antiperovskita) & Cúbico & Ferromagnético \\
\hline $\mathrm{Sr}\left(\mathrm{Fe}_{2 / 3} \mathrm{~W}_{1 / 3}\right) \mathrm{O}_{3}$ & Tetragonal & Ferrimagnético \\
\hline $\mathrm{KMnF}_{3}$ & Tetragonal & Antiferromagnético \\
\hline
\end{tabular}


Nos vamos a detener, brevemente, en la familia de los denominados compuestos III-V.

Aunque no se puede, y no se debe, elegir un grupo periódico como el más importante de la tabla de Mendeleiev, es notorio que el antiguo ${ }^{22}$ grupo IV que incluye el carbono es particularmente importante, y no solo por incluir a dicho elemento, central en la Química de la inmensa mayoría de los compuestos moleculares, -muchos de los cuales se denominan orgánicos, aunque no son, necesariamente, de origen orgánico en el sentido de natural-; la evolución de la propiedades fisicoquímicas de dichos elementos al descender en el grupo es a la vez gradual y profunda, más incluso que en sus respectivos congéneres. Y, precisamente, sus congéneres, los elementos de los grupos III y $V$, Tabla IV, combinados entre sí, forman una familia de compuestos sólidos isoestructurales con la estructura del diamante, estructura que cuando presenta dos elementos diferentes se denomina tipo estructural blenda, Figura 13a y cuyas propiedades, especialmente las electrónicas, varían de manera progresiva, lo que les hace extraordinariamente útiles. Lo mismo ocurre con las combinaciones binarias entre los elementos de los grupos II y VI. Se forma así la amplia familia de semiconductores III-Vy II-V. Tabla $\mathrm{V}$, que tienen una gran potencialidad de uso en, por ejemplo, electrónica.

\section{II.3.2. Cambio de algunos átomos de un tipo manteniendo constante el número de sitios ocupados}

En realidad, este modo de formación de compuestos nuevos lo que constituye, uno de los basamentos de la Química, va mucho más allá de la mera sustitución total de unos por otros para generar las anteriores familias. Más fructífera aun -porque da lugar a muchas más composiciones, a muchos más materiales- es la sustitución parcial, de algunos átomos por

TABLA IV: ELEMENTOS DE LOS GRUPOS II A VI DE LA TABLA PERIÓDICA

\begin{tabular}{|c|c|c|c|c|}
\hline II & III & IV & V & VI \\
\hline & B & C & N & S \\
\hline $\mathrm{An}$ & $\mathrm{Al}$ & $\mathrm{Si}$ & $\mathrm{P}$ & $\mathrm{Se}$ \\
\hline $\mathrm{Cd}$ & $\mathrm{Ga}$ & $\mathrm{Ge}$ & $\mathrm{As}$ & $\mathrm{Sb}$ \\
\hline $\mathrm{Hg}$ & $\mathrm{In}$ & $\mathrm{Sn}$ & $\mathrm{Bi}$ & $\mathrm{Te}$ \\
\hline
\end{tabular}

a)

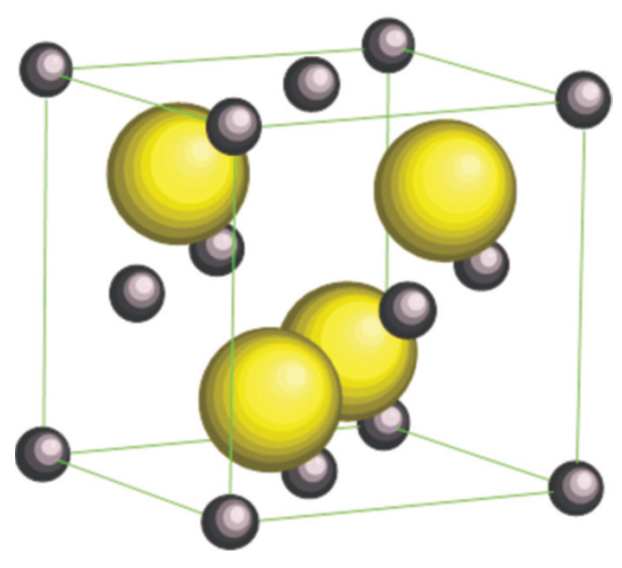

b)

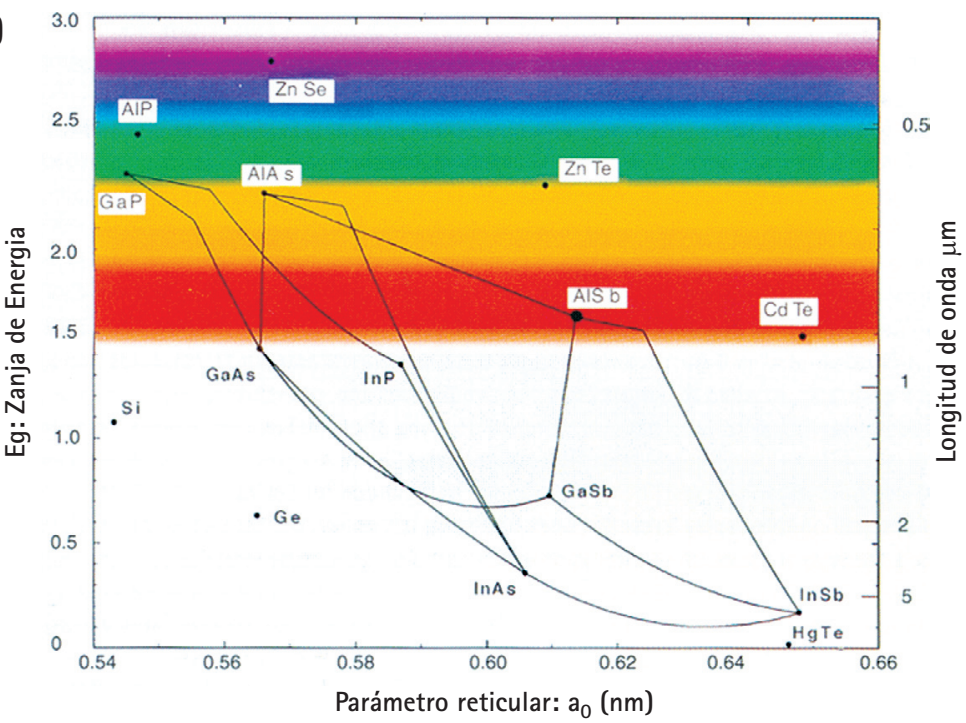

Figura 13. a) Estructura de la blenda. b) Representación de la separación entre bandas en las Familias III-V y II-VI 
TABLA V: PROPIEDADES DE LOS SEMICONDUCTORES III-V Y II-VI

\begin{tabular}{|l|l|r|r|r|}
\hline $\begin{array}{c}\text { Semi- } \\
\text { conductor }\end{array}$ & $\mathrm{a}_{0}(\mathrm{~A})$ & $\mathrm{E}_{\mathrm{g}}(\mathrm{eV})$ & \multicolumn{2}{|c|}{$\begin{array}{c}\text { Movilidad @300 K (cm²/sec) } \\
\text { electrones, huecos }\end{array}$} \\
\hline $\mathrm{Si}$ & 5.4310 & 1,11 & 1.400 & 470 \\
\hline $\mathrm{Ge}$ & 5.6461 & 0,67 & 3.900 & 1.900 \\
\hline $\mathrm{GaP}$ & 5.4506 & 2,26 & 110 & 75 \\
\hline $\mathrm{AIP}$ & 5.4625 & 2,45 & & \\
\hline $\mathrm{GaAs}$ & 5.6535 & 1,42 & 8.500 & 400 \\
\hline $\mathrm{AlAs}$ & 5.6605 & 2,17 & 280 & \\
\hline $\mathrm{InP}$ & 5.8688 & 1,35 & 5.000 & 150 \\
\hline $\mathrm{InAs}$ & 6.0584 & 0,36 & 33.000 & 460 \\
\hline $\mathrm{GaSb}$ & 6.0954 & 0,72 & 5.000 & 850 \\
\hline $\mathrm{AlSb}$ & 6.1355 & 1,58 & 900 & 450 \\
\hline $\mathrm{InSb}$ & 6.4788 & 0,17 & 80.000 & 1.250 \\
\hline $\mathrm{ZnSe}$ & 5.6676 & 2,80 & 530 & \\
\hline $\mathrm{ZnTe}$ & 6.0880 & 2,20 & 530 & 130 \\
\hline $\mathrm{CdTe}$ & 6.4816 & 1,49 & 700 & 60 \\
\hline
\end{tabular}

otros. Se forma así una solución sólida (SS) que, cuando en el proceso se mantiene constante el número de posiciones atómicas ocupadas se denomina solución sólida verdadera (SSV) y ello es tanto más fácil cuanto mayor es la similitud entre las estructuras de los componentes de la solución sólida y, en particular, entre los tamaños y los estados de oxidación de los elementos que los constituyen -lo que supone una extensión de las denominadas Reglas de Hume-Rotery.

Una solución sólida es, pues, una combinación de dos o más especies químicas que presentan la misma (o parecida) estructura y cuya composición se puede variar a voluntad variando las proporciones relativas de los componentes ${ }^{23}$. Un ejemplo típico de solución sólida es la formada por los óxidos de aluminio y cromo con la estructura corindón: $\mathrm{Al}_{2-x} \mathrm{Cr}_{x} \mathrm{O}_{3}$. La formación de soluciones sólidas permite, además, variar las propiedades de manera continua al efectuar cambios continuos en la composición. En el caso de ese óxido mixto de aluminio y cromo, la propiedad que varia de modo continuo es el volumen de la celda unidad.

En el caso de las familias III-V y II-VI, a que nos referíamos en el apartado anterior, en la formación de soluciones sólidas no solo varía de manera continua el volumen de la celda, también lo hacen la separación entre bandas, la movilidad de los portadores, la constante dieléctrica, etc. La
Figura 13b, muestra una gráfica de la evolución de la zanja para una serie de soluciones sólidas de semiconductores III-V y II-VI, superpuesta en el espectro visible. Puede observarse que la evolución de separación entre bandas zanja es, en muchos casos, pero no siempre, una función lineal de la composición y, por ende, del parámetro reticular, e.g.:

$$
\begin{gathered}
\mathrm{Al}_{x} \mathrm{Ga}_{1-x} \mathrm{As}: \mathrm{Eg}=1.42+1.24 \mathrm{x} \\
\mathrm{Ga}_{x} \operatorname{In}_{1-\mathrm{x}} \mathrm{Sb}: \mathrm{Eg}=0.172+0.165 \mathrm{x}+0.41 \mathrm{x}^{2} .
\end{gathered}
$$

Otro ejemplo muy interesante es el de la solución sólida $\mathrm{Ba}\left(\mathrm{Pb}_{1-x} \mathrm{Bi}_{\times}\right) \mathrm{O}_{3}$ formada entre los óxidos de tipo perovskita $\mathrm{BaBiO}_{3}$ y $\mathrm{BaPbO}_{3}$ que son, respectivamente un semiconductor y un metal. Las propiedades eléctricas de la SS van cambiando paulatinamente con la composición y el material se hace superconductor en el margen composicional $0,35<x<0,10$, Figura 14. El máximo en la temperatura crítica, Tc $\sim 13 \mathrm{~K}$, se alcanza para $\mathrm{x} \sim 0.10$. $Y$ esta fue durante mucho tiempo la máxima Tc para los materiales superconductores, junto con la espinela $\mathrm{LiTi}_{2} \mathrm{O}_{4}$.

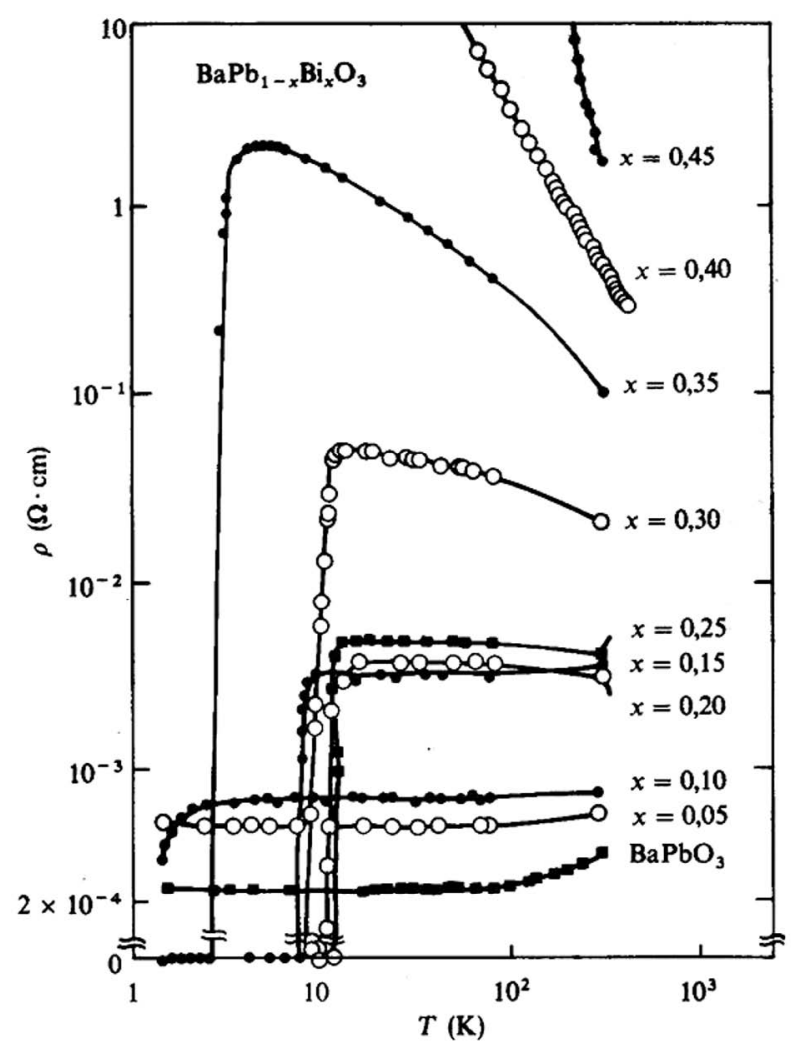

Figura 14. Evolución con la temperatura y la composición de la solución sólida $\mathrm{Ba}\left(\mathrm{Pb}_{1-x} \mathrm{Bi}_{x}\right) \mathrm{O}_{3}$

ARBOR CLXXXVII EXTRA 2011 57-79 ISSN: 0210-1963 
II.3.3. Cambio de algunos átomos de un tipo cambiando el número de sitios ocupados

Como apuntábamos más arriba, se presentan así dos tipos de situaciones: soluciones sólidas anómalas y compuestos no estequiométricos.

Soluciones sólidas anómalas: Se habla de soluciones sólidas anómalas (SSA) cuando, como resultado de la disolución, cambia el número de sitios ocupados, manteniéndose, en todo caso la estructura cristalina. Pero ese cambio puede ser por defecto -y se habla de hipoestequiometría- o por exceso -y entonces se dice que hay hiperestequiometría.

Ejemplo del primer caso, hipoestequiometría, son las soluciones sólidas que forma la zircona, con óxidos trivalentes, como los de las tierras raras y sus análogos escandio e ytrio; en el segundo caso, un ejemplo bien conocido es el de los fluoruros de calcio y de ytrio.

En la Figura $15 a$ se muestran las celdas unidad de las estructuras de $\mathrm{ZrO}_{2}$ (en realidad $\mathrm{Zr}_{4} \mathrm{O}_{8}$ ) e $\mathrm{Y}_{2} \mathrm{O}_{3} \square$ (en realidad $\mathrm{Y}_{4} \mathrm{O}_{6} \square_{2}$ ), basadas ambas en la estructura tipo fluorita. En una descripción clásica y útil, que no hay que tomar al pie de la letra, utilizando la idea de esferas compactas ${ }^{24}$, ambas estructuras se describen como un empaquetamiento cúbico de átomos metálicos, $\mathrm{Zr}$ e $\mathrm{Y}$ respectivamente, cuyos huecos tetraédricos están ocupados por ocho y seis iones oxígeno respectivamente. Al formar la solución sólida cúbica

$$
(1-x) \mathrm{ZrO}_{2}+\mathrm{x} / 2 \mathrm{Y}_{2} \mathrm{O}_{3} \square \Rightarrow \mathrm{Zr}{ }_{1-\mathrm{x}} \mathrm{Y}_{\mathrm{x}} \mathrm{O}_{2-\mathrm{x} / 2 \square \mathrm{x} / 2}
$$

en la que el símbolo $\square$ indica precisamente las posiciones oxígeno sin ocupar, al incrementarse el valor de $\mathrm{x}$ aumenta, precisamente, la concentración de $\square$; o sea que se forma una solución sólida anómala. Pues bien, estos materiales se usan en diferentes procesos en los que se quiere optimizar la conductividad iónica del oxígeno para su utilización en las denominadas pilas de combustible de óxido sólido: Una línea de investigación de gran interés y actualidad en la utilización del hidrógeno como combustible no contaminante: La hipoestequiometría generada en la formación de soluciones sólidas anómalas es pues de mucho interés e importancia en la preparación de nuevos materiales.
Un buen ejemplo del segundo caso, hiperestequiometría, también referido al propio mineral fluorita, viene representado por la ecuación química siguiente:

$$
\text { (1-x) } \mathrm{CaF}_{2}+x \mathrm{YF}_{3} \mathrm{Ca}_{1-x} \mathrm{Y}_{\mathrm{x}} \mathrm{F}_{2+\mathrm{x}} \Theta_{\mathrm{x}}
$$

en la que el símbolo $\Theta$ representa las posiciones extra ocupadas, que corresponden al centro (posición 1/2, 1/2, 1/2) de la celda unidad de la estructura, Figura $15 \mathrm{~b}$.

Compuestos no estequiométricos: Ejemplo de ambos casos, hipo-e hiperestequiometría, son también los denominados compuestos no estequiométricos(CNE). Los primeros compuestos de este tipo fueron los denominados bronces de Wolframio, descubiertos por Wholer hacia 1850 mediante la reacción de intercalación de metales alcalinos, por sublimación, sobre el trióxido de wolframio:

$$
\mathrm{xM}+\mathrm{WO}_{3} \Leftrightarrow \mathrm{M}_{\mathrm{x}}+\mathrm{WO}_{3}
$$

$Y$ es que la estructura del $W_{3}$, Figura $16 a$, que puede describirse, en una primera aproximación, como formada por octaedros que comparten todos sus vértices ${ }^{25}$, también posee un hueco central (posición 1/2, 1/2, 1/2) esta vez de geometría cubo-octaédrica con doce vértices y que es ocupado en el proceso anterior [11], parcial o totalmente ${ }^{26}$, Figura 16b. Cuando la ocupación es parcial, $0<x \leq 1$, se habla de bronces de wolframio, que son materiales cuyas propiedades varian de manera progresiva en función del grado de ocupación de dicha posición (x). Así por ejemplo, la conductividad eléctrica del semiconductor $\mathrm{WO}_{3}$ se hace metálica, lo que se atribuye a una progresiva ocupación de la banda de conducción vacía del trióxido de Wolframio -denominado "metal sin electrón"-, al recibir electrones de la oxidación del metal: En el caso de un metal alcalino

$$
M \Rightarrow M^{+}+e
$$

No es este, sin embargo, un proceso exclusivo de los alcalinos y se conocen multitud de especies químicas que pueden ser intercaladas en los huecos de la estructura.

Prácticamente todas las propiedades cambian, entre ellas el color que, al hacerse el material metálico, se hace broncíneo, de ahí la denominación de bronces ${ }^{27}$ empleada por Wohler. Así, en el caso $^{28}$ de la intercalación de zinc en este trióxido 


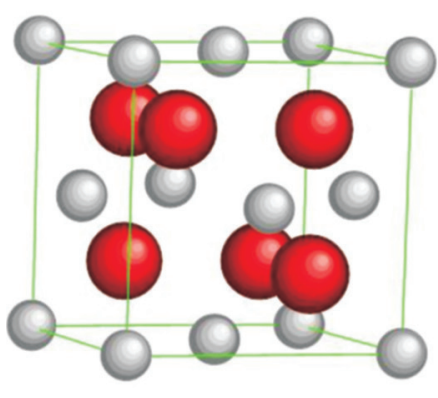

$\mathrm{Y}_{2} \mathrm{O}_{3}$ 司

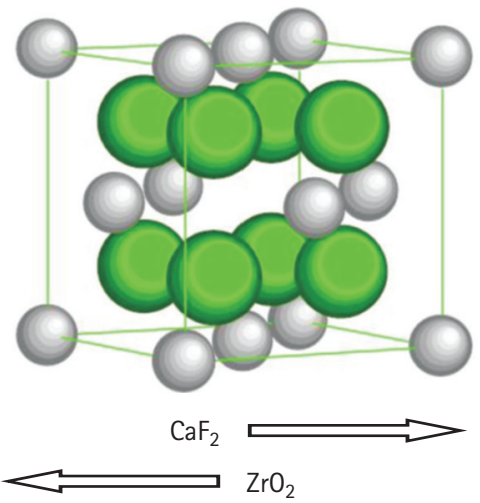

b) Hiperestequiometria

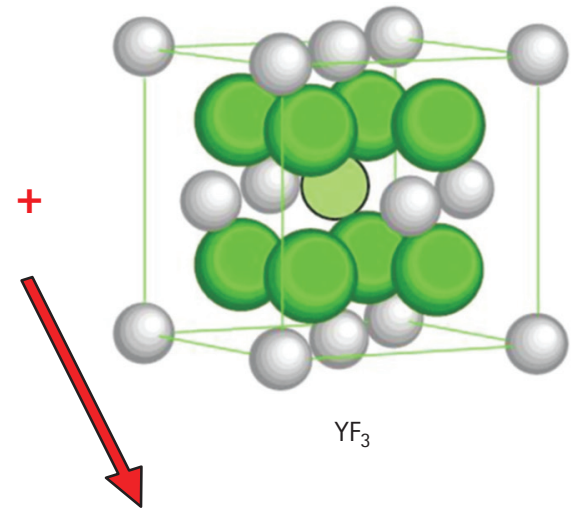

(1-x) $\mathrm{ZrO}_{2}+\mathrm{x} / 2 \mathrm{Y}_{2} \mathrm{O}_{3}$ ? $<>\mathrm{Zr}_{1-\mathrm{x}} \mathrm{Y}_{\mathrm{x}} \mathrm{O}_{2 \times / 2} \mathbf{Z}_{\mathrm{x} / 2}$

(1-x) $\mathrm{CaF}_{2}+\mathrm{x} \mathrm{YF}_{3}<>\mathrm{Ca}_{1-\mathrm{x}} \mathrm{Y}_{\mathrm{x}} \mathrm{F}_{2+\mathrm{x}} \Theta_{\mathrm{x}}$

Figura 15. Representación estructural esquemática, de la formación de soluciones sólidas anómalas (SSA) basadas en el tipo estructural fluorita

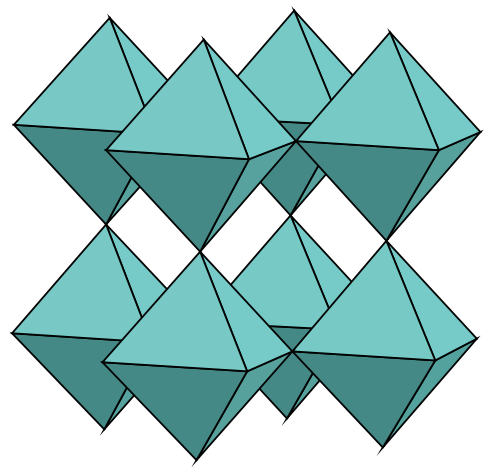

a) $\mathrm{WO}_{3}$

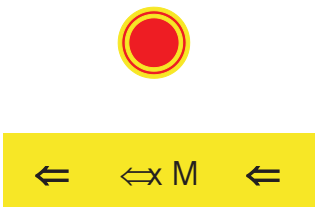

$$
\mathrm{Zn} \Rightarrow \mathrm{Zn}^{2+}+2 \mathrm{e}
$$

La conductividad eléctrica aumenta hasta cuatro órdenes de magnitud para $x=0,3$. Al mismo tiempo, el color amarillo verdoso del material original se va oscureciendo progresivamente a azul celeste, azul oscuro y violeta para esa última composición. Y ello está en línea con la evo-

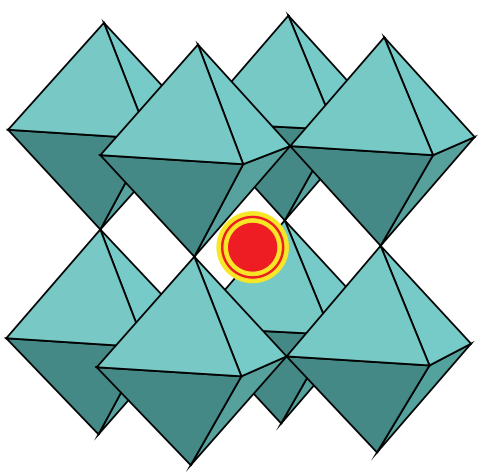

b) $\mathrm{M}_{\mathrm{x}} \mathrm{WO}_{3}$

Figura 16. Esquema de la formación de compuestos no estequiométricos por intercalación-desintercalación en los bronces de wolframio

lución observada en el caso de los bronces de sodio, con la salvedad de que, al ceder dos electrones/mol el zinc por solo uno el sodio, la composición de este de color violeta corresponde $a x=0,6$. Hay que decir también que, aunque estamos tratando en este apartado de cambios de composición manteniendo la estructura, esta cambia pasando por diferentes simetrías en el curso de la inserción,

ARBOR CLXXXVII EXTRA 2011 57-79 ISSN: 0210-1963 
hasta alcanzar la simetría cúbica. No obstante, a lo largo de este proceso paulatino, la estructura inicial formada por una red de octaedros más o menos inclinados y/o distorsionados se mantiene y podemos hablar de estructuras pseudo-cúbicas tipo perovskita, Figura 16b -véase asimismo la Figura 12.

Hemos descrito pues estos compuestos no estequiométricos como una hiperestequiometría a partir del tipo estructural trióxido de renio, característica del $\mathrm{WO}_{3}$. Pero también lo podríamos haber hecho como hipoestequiometría por desintercalación a partir del término final, $x=1$, esto es de la perovskita, $\mathrm{MWO}_{3}$, dado que el proceso es reversible:

$$
\mathrm{MWO}_{3} \Leftrightarrow \mathrm{M}_{\mathrm{x}} \mathrm{WO}_{3}+\mathrm{xM}
$$

Como último ejemplo de compuesto no estequiómétrico representante de hipo e hiperestequiometría, y que da lugar a marcados cambios en las propiedades manteniendo la estructura media, nos vamos a ocupar del importante superconductor $\mathrm{YBa}_{2} \mathrm{Cu}_{3} \mathrm{O}_{7}$, el primer material que presenta una temperatura crítica por encima de la de ebullición del nitrógeno a la presión atmosférica ${ }^{29}$.

El mencionado óxido mixto presenta, debido al orden BaY-Ba observado a lo largo del eje $\boldsymbol{c}$, una superestructura triple de la estructura perovskita, Figura $17 a$, y en la que, por razón de los estados de oxidación de los elementos que componen el material, existen dos oxígenos menos de los nueve que corresponden a tres veces $\mathrm{ABO}_{3}$; para esa composición, el material presenta una temperatura crítica de transición a la fase superconductora de 96.5 $\mathrm{K}^{30}$. Pero además, en condiciones adecuadas de presión de oxígeno y temperatura, el material puede reducirse y cambia progresivamente su estequiometría hasta alcanzar un escalón para la composición $\mathrm{YBa}_{2} \mathrm{Cu}_{3} \mathrm{O}_{7-\delta}(\delta \approx 0.5)^{31}$ lo que equivale a $\mathrm{Y}_{2} \mathrm{Ba}_{4} \mathrm{Cu}_{6} \mathrm{O}_{13}$, Figura 17b; en estas condiciones, la temperatrura crítica se sitúa en torno a los $60 \mathrm{~K}$. Si se continúa reduciendo, se llega a perder completamente el oxígeno del plano basal de la estructura Figura 17c y se alcanza la estequiometria $\mathrm{YBa}_{2} \mathrm{Cu}_{3} \mathrm{O}_{6}$, en la que el material no es superconductor. El proceso es reversible y se puede volver a la fase intermedia y a la inicial modificando las condiciones termodinámicas y oxidando el sólido: una prueba más de la influencia de la estequiometría en las propiedades del material.
Existe multitud de ejemplos de compuestos no estequiométricos, que varian su composición al variar las condiciones termodinámicas, en particular la presión de vapor de alguno de sus componentes, en particular el no metálico ya que ello es más fácil de realizar. Alguno de esto son $\mathrm{WO}_{3-x_{1}}$ $\mathrm{TiO}_{2-x}, \mathrm{UO}_{2+x}, \mathrm{M}_{\mathrm{x}} \mathrm{C}$-donde $\mathrm{C}$ puede ser grafito, fullerenos 0 nanotubos...-, etcétera.

Antes de terminar este apartado, conviene hacer una aclaración acerca de las diferencias entre soluciones sólidas anómalas (SSA) y compuestos no estequiométricos (CNE). Para ello podemos comparar un ejemplo de cada uno utilizando la misma estructura, por ejemplo la estructura de la fluorita, en las dos reacciones siguientes:

$$
\begin{gathered}
\text { SSA: (1-x) } \mathrm{CaF}_{2}+x \mathrm{YF}_{3} \Rightarrow \mathrm{Ca}_{1-\mathrm{x}} \mathrm{Y}_{\mathrm{x}} \mathrm{F}_{2+\mathrm{x}} \Theta_{\mathrm{x}} \\
\text { CNE: } \mathrm{TRH}_{2}+\mathrm{x} / 2 \mathrm{H}_{2} \Leftrightarrow \mathrm{TRH}_{2+\mathrm{x}}
\end{gathered}
$$

La primera [10], ya descrita anteriormente, da lugar a una solución sólida anómala. La segunda [15] también basada en esa estructura, con los iones de las tierras raras (TR) ligeras en los vértices y centros de las caras, y los iones oxígeno en los ocho huecos tetraédricos, consiste en la rehidruración del dihidruro de la tierra rara para dar el trihidruro en lo que se considera un proceso de no-estequiometría. En él, la estructura fluorita se contrae, por lo que aumenta la densidad, al ir entrando más ión hidruro, esta vez en los huecos octaedricos vacíos de la estructura, hasta alcanzar el límite para $\mathrm{x}=1$.

La diferencia estriba en que en el primer caso, para que la reacción se produzca tienen que difundir los iones $\mathrm{Y}(\mathrm{III})$ y $\mathrm{Ca}(\mathrm{II})$ en contracorriente en una red de iones flúor. En el segundo caso, la red metálica se mantiene y únicamente se produce un equilibrio de hidruración, entrada y salida de hidrógeno en la red.

La diferencia no es puramente académica porque se refiere a la cinética y al mecanismo de reacción, factores de importancia en la preparación de materiales. Además la reacciones del tipo de la [15] características de los compuestos no estequiométricos son reversibles y, en principio, se pueden recuperar los reactantes cambiando las condiciones termodinámicas; en esto, y en la elevada densidad volumétrica ${ }^{32}$ de hidrógeno, se basa la utilización de hidruros como materiales para la acumulación de hidrógeno 


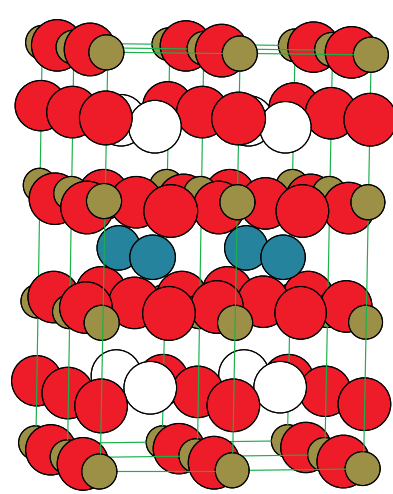

a) $\delta=0.0: \mathrm{YBa}_{2} \mathrm{Cu}_{3} \mathrm{O}_{7}$ Tc $\delta 95 \mathrm{~K}$

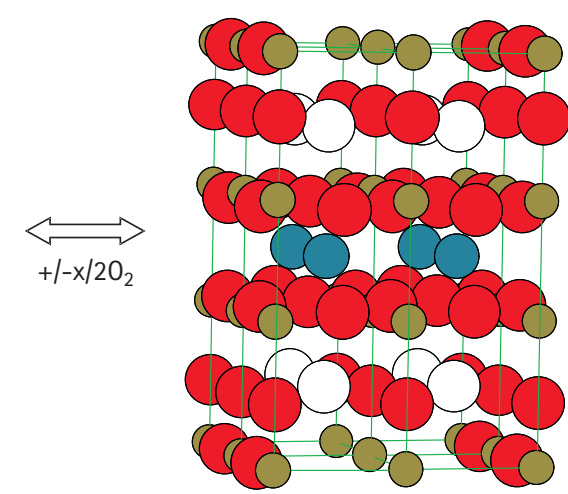

b) $\delta=0.5: \mathrm{YBa}_{2} \mathrm{Cu}_{3} \mathrm{O}_{6.5}$

$\mathrm{Tc}=\delta 60 \mathrm{~K}$

Cobre Ytrio $\bigcirc$ Bario

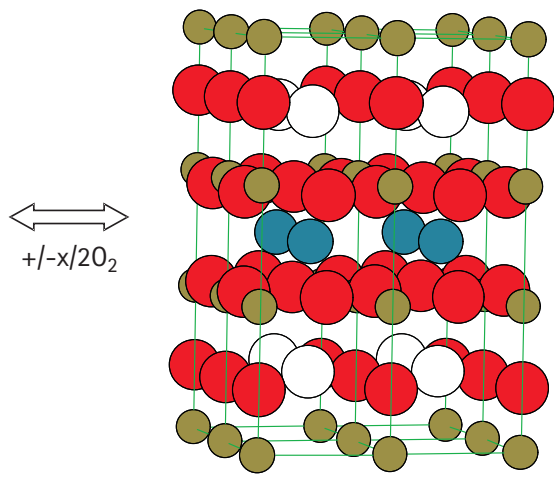

c) $\delta=1: \mathrm{YBa}_{2} \mathrm{Cu}_{3} \mathrm{O}_{6}$

$\mathrm{Tc}=0 \mathrm{~K}$

Oxígeno

Figura 17. Evolución de la estequiometría y la temperatura crítica para el superconductor $\mathrm{YBa}_{2} \mathrm{Cu}_{3} \mathrm{O}_{7-\delta}$

con vistas a su utilización en pilas de combustible y otras aplicaciones. Por el contrario, la descomposición de la solución sólida anómala en dos fases es, generalmente, un proceso más complicado.

\section{II.4. Materiales compuestos ("composite materials")}

El primer material compuesto, inventado muy probablemente en Mesopotamia -el actual Irak- estaba formado, literalmente, por cañas y barro, recibe el nombre de adobe y aun se utiliza en la construcción en zonas deprimidas de la Tierra, donde también se utiliza en forma de ladrillos.

El cemento armado y el hormigón son otros dos materiales compuestos que se también se utiliza en construcción, pero a mayor escala. Según la Real Academia de Ciencias, los materiales compuestos, que también denomina como materiales híbridos, son "cada uno de los materiales obtenidos por combinación de varias sustancias a fin de lograr propiedes que ninguna tiene por separado".

La fabricación de materiales compuestos es más compleja de lo que pudiera parecer a primera vista, por lo que la estrategia para encontrarlos también lo es. Rehecho, retrata de mezclar o combinar dos o más micro o macro- constituyentes que sean de diferente composición química y estructura, y mutuamente insolubles, y que den lugar a propiedades combinadas de las que cada uno tiene por separado o que unos refuercen las de otros.

El principio básico de los materiales compuestos es bastante simple: Si, por ejemplo, se trata de reforzar las propiedades mecánicas -que suelen ser las más beneficiadas, pero no las únicas- en este tipo de materiales, podemos pensar en utilizar fibras del mismo, de tal manera que la propagación de una grieta se circunscriba a la dimensión de la fibra. Si ensamblamos un racimo de estas fibras y las asociamos por medio de un polímero que las mantenga unidas -y al mismo proteja la superficie de un ataque exterior- sea este mecánico o químico, podremos tener un material compuesto con propiedades mecánicas mejoradas. Obviamente, hay que conseguir que ambos materiales sean compatibles y que su asociación no de lugar al deteriorote las propiedades de uno por reacción química con el(los) otro(s).

A pesar de esta dificultad, desde los parachoques de los automóviles a los cordajes de las raquetas de tenis, de los chalecos antibala a las tablas de esquiar, de las pértigas de salto a los pistones de los motores de explosión, de los muelles de válvula a las palas de helicóptero, de las 
antenas para radiocomunicaciones a los mástiles de barco y cascos para veleros marinos o los fuselajes de los veleros aéreos, los materiales compuestos van incrementando su presencia en la vida diaria y contribuyendo a una mejora continua de récords y otras hazañas.

Existen diferentes tipos de materiales compuestos y su búsqueda es, lógicamente dependiente del tipo de material compuesto y de las aplicaciones que de él se esperan. En todos ellos, sin embargo, hay un componente interior, a veces denominado refuerzo y un envolvente exterior denominado matriz. El primero de ellos constituye el soporte de las propiedades mecánicas, eléctricas, ópticas, etc. y suele estar form por fibras de los materiales más diversos como el vidrio, la alúmina, el carbón, el carburo de silicio, boro, o diferentes metales, en particular los refractarios como wolframio o molibdeno. Pero también se usan aceros austeníticos e incluso algunos materiales moleculares -"orgánicos"- de los que el más conocido es el Kevlar, un nylon aromático que, en forma de fibra trenzada o de tela, se usa como material de refuerzo y posee un límite elástico -tensión máxima reversible: sin deformación permanente- icinco veces superior al del acero a igualdad de peso...!

El material interno puede estar también en forma policristalina y también en este caso se utilizan materiales variados como grafito, carburo o nitruro de boro, alúmina y un largo etcétera.

Por lo que se refiere a la matriz, ésta suele ser polimérica, pero cada vez se descubren/inventan más materiales compuestos de matriz metálica o cerámica. Obviamente, las propiedades -y aplicaciones- de cualquiera de estos grupos son numerosísimas en cuanto a la consecución de propiedades -en particular mecánicas- a medida. Más aun, su número crece continuamente y lo hace de manera ilimitada si se hacen combinaciones con más de dos de ellos.

\section{II.5. "Otros": Azar}

No ha sido infrecuente en la Historia de la Ciencia que se produzcan descubrimientos inesperados ${ }^{33}$ y los descubrimientos de la bakelita, el teflón y hasta la penicilina fueron en gran medida inesperados. Por ello hay que estar preparado y alerta para cuando se produce el "lucky strike" o golpe de suerte...
De entre los muchos materiales interesantes descubiertos, en gran medida como fruto del azar, nos fijaremos en la denominada $\beta$-alúmina, descubierto en 1916, en el proceso Bayer de fabricación de alúmina, como punto de partida para la fabricación del aluminio y que resulta diferente de los dos óxidos habituales, $\alpha-\mathrm{Al}_{2} \mathrm{O}_{3}$, con estructura corindón y $\gamma$-alúmina, con una compleja superestructura del tipo estructural espinela. Aunque denominada $\beta$-alúmina, se sospechaba la presencia de sodio en la estructura que ha sido objeto de trabajo por parte de cristalógrafos tan ilustres como Pauling ${ }^{34}$, Bragg $^{35}$ o Beevers ${ }^{36}$ y que fue resuelta finalmente por Felsche $^{37}$. Se trata de una estructura, Figura 18, compuesta por bloques de tipo espinela separados por planos conteniendo sodio y oxígeno en pequeña cantidad, lo que significa dos cosas importantes. La primera es que

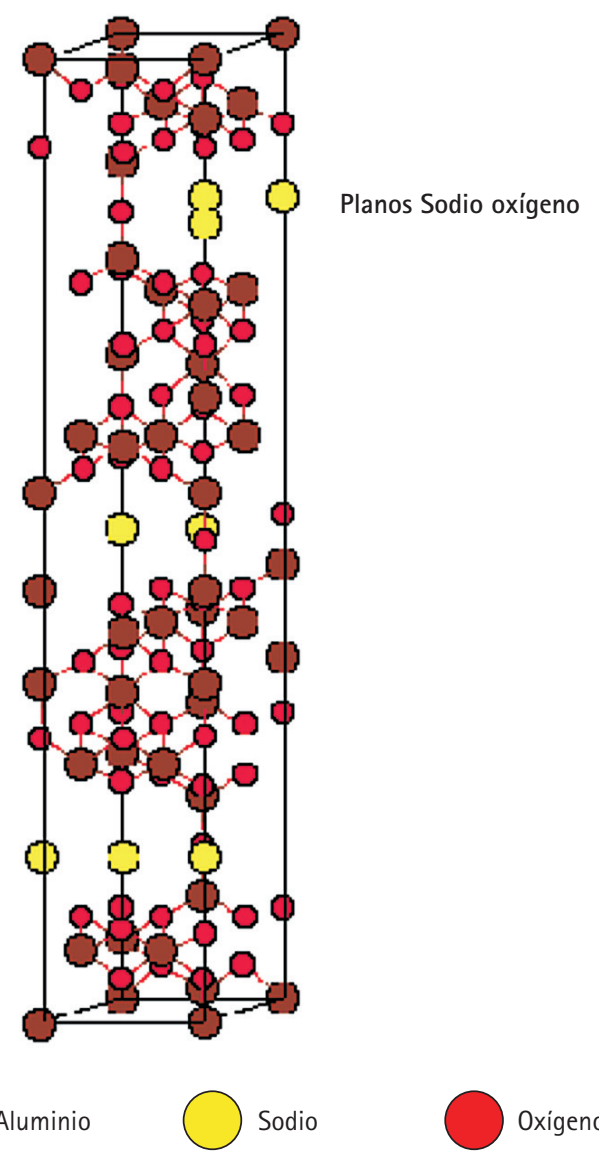

Figura 18. Estructura de la $\beta$-Alúmina 
la composición no es alúmina pura, sino $\mathrm{Na}_{2} \mathrm{Al}_{22} \mathrm{O}_{34}<>$ $\mathrm{Na}_{2} \mathrm{O} \cdot 11 \mathrm{Al}_{2} \mathrm{O}_{3}$. La segunda que eso le hace ser un muy buen conductor iónico de sodio lo que ha dado lugar a un nuevo tipo de materiales denominados sucesivamente "Superconductores lónicos" (Ionic Superconductors), "Conductores lónicos Rápidos" (Fast ionic conductors) que se debe convertir en Conductores lónicos Óptimos y alguna otra denominación. Además, esto supuso el punto de partida de, nada menos que, una nueva disciplina en Química del Estado Sólido: Solid State Ionics- literalmente "Ionica del Estado Sólido".

No es este el único material de este tipo y existen diferentes variedades estructurales que dan lugar a diferentes familias. La más próxima es la de las beta-alúminas que se usan en baterias de estado sólido en las que el electrolito es, efectivamente sólido: una de las diferentes beta alúminas y los electrodos líquidos: sodio y azufre que en el proceso de oxidación de aquel producen sulfuro sódico en proceso reversible. Estas baterias se desarrollaron en vista de su utilización en automoción durante la crisis del petróleo de los años setenta del siglo $X X$, y se llevó a cabo un gran esfuerzo en investigación por parte de las compañías automovilistas.

Una familia estrechamente relacionada con la anterior es la de las ferritas hexagonales o hexaferritas, materiales en los que sodio y aluminio están sustituidos por diferentes cationes, en particular cationes magnéticos como hierro, cobalto y níquel y que constituyen la base de los imanes domésticos de gran interés mercantil.

\section{A modo de Conclusión}

Para terminar -iprovisionalmente!- tan apasionante tema y al que en este relativamente limitado espacio, quisieramos señalar otra estrategia para buscar materiales y que no hemos abordado en esta ocasión. Se trata de la estrategia que utiliza la Naturaleza para alcanzar el material óptimo para cada aplicación concreta. Se abre así el mundo de los biominerales, en el que una combinación generalmente sutil y compleja de un mineral, como la calcita, y una proteína dan lugar a un sólido tan perfecto y útil como la concha de los moluscos. Lo mismo podríamos decir de los dientes, las plumas o los huesos de los animales, o los productos de su metabiolismo como la cera o la seda.

Materiales importantes son también los que proceden del mundo vegetal, como la madera o las fibras. Otra manera de llegar a obtener sólidos útiles con alto valor añadido, esto es: otra estrategia para la búsqueda de materiales es, pues, imitar a la Naturaleza que en unos cuantos millones de años de prueba y error ha llegado a construir un mundo material en sí mismo perfecto y al que debemos imitar, pero no distorsionar.

La Historia, decíamos al principio, está llena de ejemplos de materiales que nos han hecho la vida más agradable pero lo más agradable es pensar que teniendo en cuenta la vitalidad actual de la Ciencia de Materiales, esta continuará asombrándonos y, a través de los nuevos sólidos útiles que continuarán descubriéndose, tendremos cada vez más posibilidades de disfrutar de nuestro entorno natural.

\section{NOTAS}

1 En sentido antropológico: el hombre que se interesa por cosas prácticas.

2 Digamos en este contexto que, en realidad, un objeto hecho con un material si que se cansa-recordemos-, los aviones Comet 0 , de un modo ciertamente más lírico, que "... por un clavo se perdió una herradura, por una herradura un caballo, por un caballo una batalla...", pero los clavos se han seguido usando para herrar a los caballos... ide materiales cada vez mejores!

3 Justin Moresco GIGAOM, Jun. 5, 2009.

4 BCC Research: SMCO43B - November 2010.

5 J. B. Goodenough: Magnetism and the chemical bond, John Wiley, Nueva York 1963.

6 D. M. Adams, Inorganic Solids, Wiley, 1974.

7 A. R. West, Solid State Chemistry, Wiley, 1998, Capítulo 13. 
8 La fase hexagonal presenta una deficiencia de oxígeno, $\mathrm{BaTiO}_{3-x \prime}$ que depende de las condiciones termodinámicas: presión de vapor de oxígeno y temperatura.

9 A. Vegas et al., Acta Cryst B(2001) 57(2), p. 151.

10 R. J. Hazen, The new alchemists, Times Books, 1994.

11 En filosofía de la Ciencia, el concepto de paradigma recoge la idea de que, en un determinado campo del saber, existen una serie de criterios aceptados por la mayoría de los que en ese campo trabajan.

12 R. Newnham, Structure Properties Relations, Springer Verlag, Berlín 1975.

13 Se llega, en ocasiones, a decir que es la estructura que se observa con un microscopio de más de 25 aumentos... Pero, obviamente, no es un criterio riguroso.

14 MarSol Martín González, Tesis Doctoral. Universidad Complutense, Madrid 1998.

15 M. ${ }^{\text {a }}$ Antonia Señarís Rodríguez, Tesis Doctoral, Universidad Complutense, Madrid 1993.

16 Alejandro Várez Álvarez, Tesis Doctoral. Universidad Complutense, Madrid 1993.

17 A. F. Wells, Structural Inorganic Chemistry, Oxford University Press, 4. ${ }^{\text {a }}$ Edición, Oxford 1972.

18 El término de estequiometría, deri-

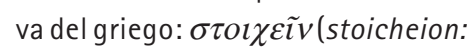
elemento) y $\mu \varepsilon ́ \tau \rho o v$ (metron: medida) y refleja las relaciones numéricas cuantitativas existentes entre los coeficientes de reactantes y productos en una reacción química $y$, en nuestro caso las que se presentan entre los diferentes elementos que forman la especie: e.g. en el mineral corindón, $\mathrm{Al}_{2} \mathrm{O}_{{ }_{3}}$, la estequiometría es 2:3. En ge- neral, la estequiometría se refiere a las relaciones entre los elementos menos y más electronegativos, como en el caso anterior. Pero para un compuesto ternario hay tres estequiometrías: e.g. en el mineral espinela, $\mathrm{MgAl}_{2} \mathrm{O}_{4}$ serian: $\mathrm{Mg} / 0$ : $1 / 4 ; A l / 0: 1: 2$ y Mg/Al: 1:2. Aunque estas relaciones se dan entre números enteros sencillos, como sugieren las leyes clásicas de la Química de Proust y Dalton, existen multitud de especies químicas en que no lo son. Se trata de sólidos no moleculares, que pueden variar su composición en las condiciones termodinámicas adecuadas, por los que sus estequiometrías son variables en dichas condiciones y no corresponden necesariamente a números sencillos.

19 L. Smart and E. Moore, Solid State Chemistry: An introduction, Chapman Et Hall, Londres 83. Edición (2008).

20 Garnets (Garnets: referencia 17; página 811).

21 A. Goldman, Modern Ferrite Technology, Springer Verlag, 2006.

22 En anteriores versiones de la Tabla Periódica se subdividian los grupos en a y $b$, siendo estos últimos los elementos de transición que, debido a la ocupación paulatina de los orbitales $d$ al aumentar el número atómico, presentan una química sui generis. En ella, pues, los grupos de boro, carbono y oxígeno eran, respectivamente los $3 a$, $4 a$ y $5 a$. Al incluirse los elementos de transición en las posiciones correlativas correspondientes a sus números atómicos, dichos grupos son ahora los 13,14 y 15 , respectivamente.

23 Desde un punto de vista básico, una solución sólida es análoga a una disolución líquida, no obstan- te, para conseguir su formación es necesaria la utilización de altas temperaturas $-\mathrm{y}$, a veces, también de altas presiones-; no obstante, no suele ser necesario alcanzar la temperatura de fusión de los componentes.

24 A. F. Wells, Referencia 17.

25 Si los octaedros son regulares se obtiene una estructura cúbica que corresponde al tipo estructural trióxido de renio, $\mathrm{ReO}_{3}$.

26 Cuando la ocupación del hueco central es total, i.e. para $x=1$, se trata del tipo estructural perovskita, auténtico laboratorio teórico y experimental y uno de los más importantes e interesantes de la Química y la Física del Estado Sólido, de la Ciencia de Materiales; y aun de las Ciencias de la Tierra por ser la estructura mineral más abundante en el manto terrestre.

27 Generalmente se entiende por bronce a una aleación metálica de cobre y estaño que, a menudo, lleva otros componentes para modificar de alguna manera sus propiedades.

28 A. Martínez de la Cruz, F. García-Alvarado, E. Morán, and M. A. AlarioFranco, J. Mater. Chem., 1998, 8(8), 1805-1807.

29 M. Á. Alario y Franco y J. L. Vicent: Supereconductividad, Eudema, Madrid 1991, capítulo 9.

30 F. G. Alvarado, E. Morán, M.Á. AlarioFranco et al., Solid State Communications (1988).

31 C. Chaillout, M. Á. Alario-Franco et al., Solid State Communications 65, 287 (1988).

32 El contenido volumétrico de hidrógeno en el hidruro de $\mathrm{LaNi}_{5}$ es de 130 $\mathrm{Kh} \mathrm{H} / \mathrm{m}^{3}$, mientras que el hidrógeno líquido solo alcanza los 70 -además ha de estar comprimido e incluso a bajas temperaturas. Sin embargo. La 
capacidad gravimétrica es mucho menor: en el citado $\mathrm{LaNi}_{5}$ es de solo un $1,4 \%$.

33 R. M. Robergs, Descubrimientos accidentales de la Ciencia, Alianza, Madrid 1992.
34 S. B. Hendricks and L. Pauling, Z. Kristallogr., 64, 303-308 (1927).

35 W. L. Bragg, C. Gottfried and J. West, Z. Kristallogr. 77, 255-274 (1931).

S. B. Hendricks and L. Pauling, Z. Kristallogr. 64, 303-308 (1927).
H. Saalfeld (1956), Z. anorg. allg. Chern. 286, 174-179.

36 C. A. Beevers y M. A. S. Ross, Z. Kristallogr. 97, 59-66 (1937).

37 J.Felsche,ZeitschriftfUrKl'istallographie, Bd. 127, S. 94-100 (1968). 